\title{
Specht filtrations and tensor spaces for the Brauer algebra
}

\author{
Jun Hu
}

Received: 4 January 2007 / Accepted: 9 October 2007 / Published online: 23 October 2007

(C) Springer Science+Business Media, LLC 2007

\begin{abstract}
Let $m, n \in \mathbb{N}$. In this paper we study the right permutation action of the symmetric group $\mathfrak{S}_{2 n}$ on the set of all the Brauer $n$-diagrams. A new basis for the free $\mathbb{Z}$-module $\mathfrak{B}_{n}$ spanned by these Brauer $n$-diagrams is constructed, which yields Specht filtrations for $\mathfrak{B}_{n}$. For any $2 m$-dimensional vector space $V$ over a field of arbitrary characteristic, we give an explicit and characteristic-free description of the annihilator of the $n$-tensor space $V^{\otimes n}$ in the Brauer algebra $\mathfrak{B}_{n}(-2 m)$. In particular, we show that it is a $\mathfrak{S}_{2 n}$-submodule of $\mathfrak{B}_{n}(-2 m)$.
\end{abstract}

Keywords Brauer algebra $\cdot$ Symmetric group $\cdot$ Tensor space

\section{Introduction}

Let $x$ be an indeterminate over $\mathbb{Z}$. The Brauer algebra $\mathfrak{B}_{n}(x)$ over $\mathbb{Z}[x]$ is a unital associative $\mathbb{Z}[x]$-algebra with generators $s_{1}, \cdots, s_{n-1}, e_{1}, \cdots, e_{n-1}$ and relations (see [16]):

$$
\begin{gathered}
s_{i}^{2}=1, \quad e_{i}^{2}=x e_{i}, \quad e_{i} s_{i}=e_{i}=s_{i} e_{i}, \quad \forall 1 \leq i \leq n-1, \\
s_{i} s_{j}=s_{j} s_{i}, \quad s_{i} e_{j}=e_{j} s_{i}, \quad e_{i} e_{j}=e_{j} e_{i}, \quad \forall 1 \leq i<j-1 \leq n-2, \\
s_{i} s_{i+1} s_{i}=s_{i+1} s_{i} s_{i+1}, \quad e_{i} e_{i+1} e_{i}=e_{i}, \quad e_{i+1} e_{i} e_{i+1}=e_{i+1}, \quad \forall 1 \leq i \leq n-2, \\
s_{i} e_{i+1} e_{i}=s_{i+1} e_{i}, \quad e_{i+1} e_{i} s_{i+1}=e_{i+1} s_{i}, \quad \forall 1 \leq i \leq n-2 .
\end{gathered}
$$

$\mathfrak{B}_{n}(x)$ is a free $\mathbb{Z}[x]$-module with rank $(2 n-1) \cdot(2 n-3) \cdots 3 \cdot 1$. For any commutative $\mathbb{Z}[x]$-algebra $R$ with $x$ specialized to $\delta \in R$, we define $\mathfrak{B}_{n}(\delta)_{R}:=R \otimes_{\mathbb{Z}[x]} \mathfrak{B}_{n}(x)$.

Research supported by National Natural Science Foundation of China (Project 10401005) and by the Program NCET. The author is very grateful to the referee for careful reading and many corrections.

J. Hu (凶)

Department of Applied Mathematics, Beijing Institute of Technology, Beijing 100081, China

e-mail: junhu303@yahoo.com.cn 
This algebra was first introduced by Richard Brauer (see [2]) when he studied how the $n$-tensor space $V^{\otimes n}$ decomposes into irreducible modules over the orthogonal group $O(V)$ or the symplectic group $S p(V)$, where $V$ is an orthogonal vector space or a symplectic vector space. In Brauer's original formulation, the algebra $\mathfrak{B}_{n}(x)$ was defined as the complex linear space with basis the set $\mathrm{Bd}_{n}$ of all the Brauer $n$-diagrams, graphs on $2 n$ vertices and $n$ edges with the property that every vertex is incident to precisely one edge. If we arrange the vertices in two rows of $n$ each, the top and bottom rows, and label the vertices in each row of a $n$-diagram by the indices $1,2, \cdots, n$ from left to right, then the generator $s_{i}$ corresponds to the $n$-diagram with edges connecting vertices $i$ (respectively, $i+1$ ) on the top row with $i+1$ (respectively, $i$ ) on bottom row, and all other edges are vertical, connecting vertex $k$ on the top and bottom rows for all $k \neq i, i+1$. The generator $e_{i}$ corresponds to the $n$-diagram with horizontal edges connecting vertices $i, i+1$ on the top and bottom rows, and all other edges are vertical, connecting vertex $k$ on the top and bottom rows for all $k \neq i, i+1$. The multiplication of two Brauer $n$-diagrams is defined as follows. We compose two diagrams $D_{1}, D_{2}$ by identifying the bottom row of vertices in the first diagram with the top row of vertices in the second diagram. The result is a graph, with a certain number, $n\left(D_{1}, D_{2}\right)$, of interior loops. After removing the interior loops and the identified vertices, retaining the edges and remaining vertices, we obtain a new Brauer $n$-diagram $D_{1} \circ D_{2}$, the composite diagram. Then we define $D_{1} \cdot D_{2}=x^{n\left(D_{1}, D_{2}\right)} D_{1} \circ D_{2}$. In general, the multiplication of two elements in $\mathfrak{B}_{n}(x)$ is given by the linear extension of a product defined on diagrams. For example, let $d$ be the following Brauer 5-diagram.

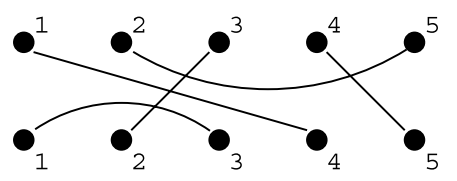

Let $d^{\prime}$ be the following Brauer 5-diagram.

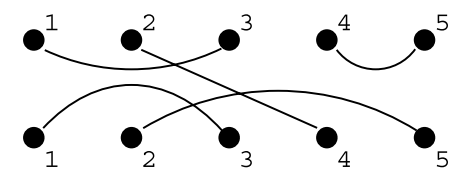

Then $d d^{\prime}$ is equal to

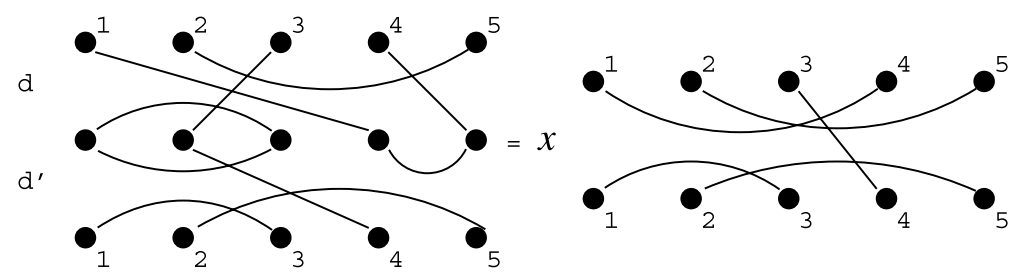


Note that the subalgebra of $\mathfrak{B}_{n}(x)$ generated by $s_{1}, s_{2}, \cdots, s_{n-1}$ is isomorphic to the group algebra of the symmetric group $\mathfrak{S}_{n}$ over $\mathbb{Z}[x]$.

The Brauer algebra as well as its quantization (now called the Birman-WenzlMurakami algebra) has been studied in a number of papers, e.g., [2-4, 6, 11, 16-18, 22, 23, 29, 35]. The walled Brauer algebra (which is a variant of the Brauer algebra, see [5]) is also studied in the recent preprint [10]. We are mainly interested in the Schur-Weyl duality between symplectic groups and certain specialized Brauer algebras, which we now recall. Let $K$ be an arbitrary infinite field. Let $m, n \in \mathbb{N}$. Let $V$ be a $2 m$-dimensional $K$-vector space equipped with a non-degenerate skew-symmetric bilinear form (, ). Then (see [19], [15, Section 4]) the symplectic similitude group (respectively, the symplectic group) relative to $($, ) is

$$
\begin{gathered}
G S p(V):=\left\{\begin{array}{l}
g \in G L(V) \mid \begin{array}{c}
\exists d \in K \text { with } d \neq 0, \text { such that } \\
(g v, g w)=d(v, w), \forall v, w \in V
\end{array}
\end{array}\right\} \\
(\text { respectively, } S p(V):=\{g \in G L(V) \mid(g v, g w)=(v, w), \forall v, w \in V\}) .
\end{gathered}
$$

The symplectic similitude group and symplectic group $S p(V)$ act naturally on $V$ from the left-hand side, and hence on the $n$-tensor space $V^{\otimes n}$. This left action on $V^{\otimes n}$ is centralized by certain specialized Brauer algebra, which we recall as follows. Let $\mathfrak{B}_{n}(-2 m):=\mathbb{Z} \otimes_{\mathbb{Z}[x]} \mathfrak{B}_{n}(x)$, where $\mathbb{Z}$ is regarded as $\mathbb{Z}[x]$-algebra by specifying $x$ to $-2 m$. Let $\mathfrak{B}_{n}(-2 m)_{K}:=K \otimes_{\mathbb{Z}} \mathfrak{B}_{n}(-2 m)$, where $K$ is regarded as $\mathbb{Z}$-algebra by sending each integer $a$ to $a \cdot 1_{K}$. Then there is a right action of the specialized Brauer algebra $\mathfrak{B}_{n}(-2 m)_{K}$ on the $n$-tensor space $V^{\otimes n}$ which commutes with the above left action of $G S p(V)$. We recall the definition of this action as follows. Let $\delta_{i j}$ denote the value of the usual Kronecker delta. For any $1 \leq i \leq 2 m$, we set

$$
i^{\prime}:=2 m+1-i
$$

We fix an ordered basis $\left\{v_{1}, v_{2}, \cdots, v_{2 m}\right\}$ of $V$ such that

$$
\left(v_{i}, v_{j}\right)=0=\left(v_{i^{\prime}}, v_{j^{\prime}}\right), \quad\left(v_{i}, v_{j^{\prime}}\right)=\delta_{i j}=-\left(v_{j^{\prime}}, v_{i}\right), \quad \forall 1 \leq i, j \leq m .
$$

For any $i, j \in\{1,2, \cdots, 2 m\}$, let

$$
\epsilon_{i, j}:= \begin{cases}1 & \text { if } j=i^{\prime} \text { and } i<j \\ -1 & \text { if } j=i^{\prime} \text { and } i>j \\ 0 & \text { otherwise }\end{cases}
$$


The right action of $\mathfrak{B}_{n}(-2 m)$ on $V^{\otimes n}$ is defined on generators by

$$
\begin{aligned}
& \left(v_{i_{1}} \otimes \cdots \otimes v_{i_{n}}\right) s_{j} \\
& \quad:=-\left(v_{i_{1}} \otimes \cdots \otimes v_{i_{j-1}} \otimes v_{i_{j+1}} \otimes v_{i_{j}} \otimes v_{i_{j+2}} \otimes \cdots \otimes v_{i_{n}}\right) \\
& \left(v_{i_{1}} \otimes \cdots \otimes v_{i_{n}}\right) e_{j} \\
& \quad:=\epsilon_{i_{j}, i_{j+1}} v_{i_{1}} \otimes \cdots \otimes v_{i_{j-1}} \otimes\left(\sum_{k=1}^{m}\left(v_{k^{\prime}} \otimes v_{k}-v_{k} \otimes v_{k^{\prime}}\right)\right) \otimes v_{i_{j+2}} \otimes \cdots \otimes v_{i_{n}} .
\end{aligned}
$$

Let $\varphi, \psi$ be the following natural $K$-algebra homomorphisms.

$$
\begin{aligned}
& \varphi:\left(\mathfrak{B}_{n}(-2 m)_{K}\right)^{o p} \rightarrow \operatorname{End}_{K}\left(V^{\otimes n}\right), \\
& \psi: K G \operatorname{Sp}(V) \rightarrow \operatorname{End}_{K}\left(V^{\otimes n}\right) .
\end{aligned}
$$

Let $k$ be a positive integer. A composition of $k$ is a sequence of nonnegative integers $\lambda=\left(\lambda_{1}, \lambda_{2}, \cdots\right)$ with $\sum_{i>1} \lambda_{i}=k$. A composition $\lambda=\left(\lambda_{1}, \lambda_{2}, \cdots\right)$ of $k$ is said to be a partition if $\lambda_{1} \geq \lambda_{2} \geq \cdots$. In this case, we write $\lambda \vdash k$. The conjugate of $\lambda$ is defined to be the partition $\lambda^{\prime}=\left(\lambda_{1}^{\prime}, \lambda_{2}^{\prime}, \cdots\right)$, where $\lambda_{j}^{\prime}:=\#\left\{i \mid \lambda_{i} \geq j\right\}$ for $j=1,2, \cdots$. For any partition $\lambda=\left(\lambda_{1}, \lambda_{2}, \cdots\right)$, we use $\ell(\lambda)$ to denote the largest integer $t$ such that $\lambda_{t} \neq 0$.

Lemma 1.1 ([2-4, 11, 32]) (1) The natural left action of $G S p(V)$ on $V^{\otimes n}$ commutes with the right action of $\mathfrak{B}_{n}(-2 m)$.

(2) if $K$ is an infinite field, then

(a) $\varphi\left(\mathfrak{B}_{n}(-2 m)_{K}\right)=\operatorname{End}_{K G S p(V)}\left(V^{\otimes n}\right)=\operatorname{End}_{K S p(V)}\left(V^{\otimes n}\right)$,

(b) $\psi(K G S p(V))=\operatorname{End}_{\mathfrak{B}_{n}(-2 m)_{K}}\left(V^{\otimes n}\right)$,

(3) if $K$ is an infinite field and $m \geq n$ then $\varphi$ is injective, and hence an isomorphism onto $\operatorname{End}_{K G S p(V)}\left(V^{\otimes n}\right)$,

(4) if $K=\mathbb{C}$, then there is a decomposition of $V^{\otimes n}$ as a direct sum of irreducible $\mathbb{C} G S p(V)-\mathfrak{B}_{n}(-2 m)_{\mathbb{C}}$ bimodules

$$
V^{\otimes n}=\bigoplus_{\substack{f=0 \\ \lambda \vdash n-2 f \\ \ell(\lambda) \leq m}}^{[n / 2]} \Delta(\lambda) \otimes D\left(\lambda^{\prime}\right),
$$

where $\Delta(\lambda)$ (respectively, $D\left(\lambda^{\prime}\right)$ ) denotes the irreducible $\mathbb{C} G S p(V)$-module (respectively, the irreducible $\mathfrak{B}_{n}(-2 m)_{\mathbb{C}}$-module) corresponding to $\lambda$ (respectively, corresponding to $\left.\lambda^{\prime}\right)$.

Historically, the above results in the case of $K=\mathbb{C}$ are proved in [2, 3] and [4]. For arbitrary infinite field $K, 2$ ) and 3) are proved in [11] and [32].

Now there is a natural question, that is, how can one describe the kernel of the homomorphism $\varphi$ ? This question is closely related to invariant theory: see [7]. By [11, Theorem 1.2], we know that the kernel of the homomorphism $\varphi$ has a rigid 
structure in the sense that the dimension of $\operatorname{Ker} \varphi$ does not depend on the choice of the infinite field $K$, and it is actually defined over $\mathbb{Z}$. Note that in the case of SchurWeyl duality between general linear group and symmetric group [7, 8, 33, 34], or more generally, between the type $A$ quantum group and the type $A$ Iwahori-Hecke algebra [14, 24], the kernel of the corresponding homomorphism has already been explicitly determined in [14] in terms of the Kazhdan-Lusztig basis and in [20] in terms of the Murphy basis. In this paper, we completely answer the above question by explicitly constructing an integral basis for the kernel of the homomorphism $\varphi$. Our description of $\operatorname{Ker} \varphi$ involves a study of the permutation action of the symmetric group $\mathfrak{S}_{2 n}$ on the Brauer algebra $\mathfrak{B}_{n}(x)$. Such a permutation action was previously noted in [17]. We construct a new integral basis for this Brauer algebra, which yields an integral Specht filtration of this Brauer algebra by right $\mathfrak{S}_{2 n}$-modules. The kernel of $\varphi$ is just one of the $\mathfrak{S}_{2 n}$-submodules appearing in this filtration. In particular, it turns out that $\operatorname{Ker} \varphi$ is in fact a $\mathfrak{S}_{2 n}$-submodule of $\mathfrak{B}_{n}(-2 m)$. The main results of this paper are presented in Theorem 2.11, Theorem 2.13 and Theorem 3.4. It would be interesting to compare the new integral basis we obtained in this paper with the canonical basis for $\mathfrak{B}_{n}(x)$ constructed in [17]. It would also be interesting to see how the description of $\operatorname{Ker} \varphi$ we give here can be generalized to the quantized case, i.e., the case of Schur-Weyl duality between the quantized enveloping algebra associated to the symplectic Lie algebra $s p_{2 m}$ and a certain specialized Birman-Wenzl-Murakami algebra (see [9]).

\section{The $\mathfrak{S}_{2 n}$-action on $\mathfrak{B}_{n}(x)$}

In this section, we shall first recall (cf. [17]) the right permutation action of the symmetric group $\mathfrak{S}_{2 n}$ on the set $\mathrm{Bd}_{n}$. Then we shall construct a new $\mathbb{Z}$-basis for the resulting right $\mathfrak{S}_{2 n}$-module, which yields filtrations of $\mathfrak{B}_{n}(x)$ by right $\mathfrak{S}_{2 n}$-modules. Certain submodules occurring in this filtration will play a central role in the next section.

For any fixed-point-free involution $\sigma$ in the symmetric group $\mathfrak{S}_{2 n}$, the conjugate $w^{-1} \sigma w$ of $\sigma$ by $w \in \mathfrak{S}_{2 n}$ is still a fixed-point-free involution. Therefore, we have a right action of the symmetric group $\mathfrak{S}_{2 n}$ on the set of all the fixed-point-free involutions in $\mathfrak{S}_{2 n}$. Note that the set $\mathrm{Bd}_{n}$ of Brauer $n$-diagrams can be naturally identified with the set of fixed-point-free involutions in $\mathfrak{S}_{2 n}$ as explained below. Hence we get (cf. [17]) a right permutation action of the symmetric group $\mathfrak{S}_{2 n}$ on the set $\mathrm{Bd}_{n}$ of all the Brauer $n$-diagrams. We use " $*$ " to denote this right permutation action.

We shall adopt a new labeling of the vertices in each Brauer diagram. Namely, for each Brauer $n$-diagram $D$, we shall label the vertices in the top row of $D$ by odd integers $1,3,5, \cdots, 2 n-1$ from left to right, and label the vertices in the bottom row of $D$ by even integers $2,4,6, \cdots, 2 n$ from left to right. This way of labeling is more convenient when studying the permutation action from $\mathfrak{S}_{2 n}$. We shall keep this way of labeling from this section until the end of Section 3, and we shall recover our original way of labeling only in Section 4. Let us look at an example. Suppose $n=4, s_{1} s_{7} s_{6}=(1,2)(6,7,8)$ is a permutation in $\mathfrak{S}_{8}$. Let $D$ be the following Brauer 4-diagram. 


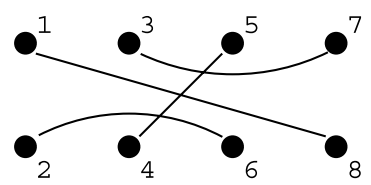

We first identify $D$ with following diagram with 8 vertices.

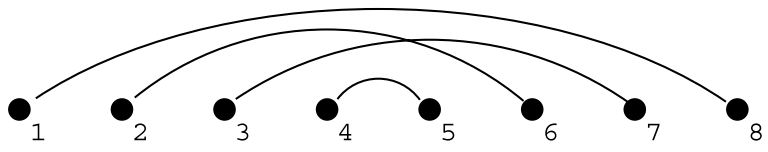

Then $D *\left(s_{1} s_{7} s_{6}\right)$ can be computed in the following way.

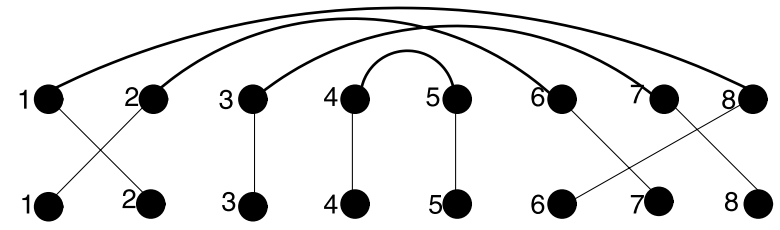

Finally, $D *\left(s_{1} s_{7} s_{6}\right)$ is equal to the following Brauer 4-diagram.

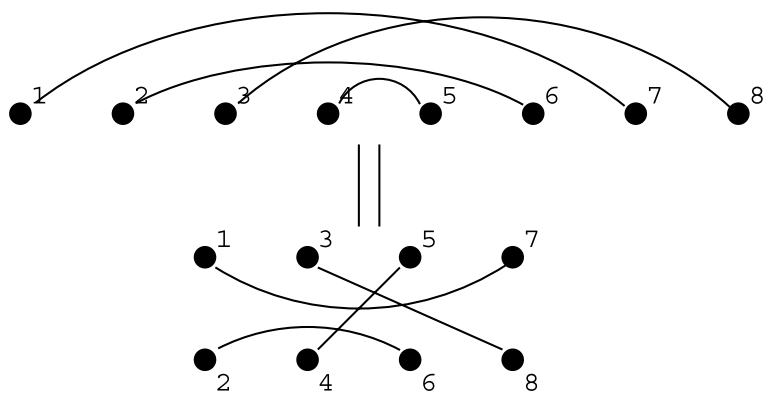

We use $\beta$ to denote the natural identification of $\mathrm{Bd}_{n}$ with the set of fixed-point-free involutions in $\mathfrak{S}_{2 n}$. For any $w \in \mathfrak{S}_{2 n}$ and any $D \in \mathrm{Bd}_{n}, D * w=\beta^{-1}\left(w^{-1} \beta(D) w\right)$.

For any commutative $\mathbb{Z}$-algebra $R$, we use $\mathfrak{B}_{n, R}$ to denote the free $R$-module spanned by $\mathrm{Bd}_{n}$. Then $\mathfrak{B}_{n, R}$ becomes a right $R\left[\mathfrak{S}_{2 n}\right]$-module. Let $\mathfrak{B}_{n}:=\mathfrak{B}_{n, \mathbb{Z}}$. Clearly, there is a canonical isomorphism $\mathfrak{B}_{n, R} \cong R \otimes_{\mathbb{Z}} \mathfrak{B}_{n}$, which is also a right $R\left[\mathfrak{S}_{2 n}\right]$-module isomorphism. Taking $R=\mathbb{Z}[x]$, we deduce that the Brauer algebra $\mathfrak{B}_{n}(x)$ becomes a right $\mathbb{Z}[x]\left[\mathfrak{S}_{2 n}\right]$-module. Similarly, the specialized Brauer algebra $\mathfrak{B}_{n}(-2 m)$ becomes a right $K\left[\mathfrak{S}_{2 n}\right]$-module. 
For any integer $i$ with $1 \leq i \leq 2 n$, we define

$$
\gamma(i):= \begin{cases}i+1, & \text { if } i \text { is odd } \\ i-1, & \text { if } i \text { is even }\end{cases}
$$

Then $\gamma$ is an involution on $\{1,2, \cdots, 2 n\}$. It is well-known that the subgroup

$$
\left\{w \in \mathfrak{S}_{2 n} \mid(\gamma(a)) w=\gamma(a w) \text { for any integer } a \text { with } 1 \leq a \leq 2 n\right\}
$$

is isomorphic to the wreath product $\mathbb{Z}_{2} 2 \mathfrak{S}_{n}$ of $\mathbb{Z}_{2}$ and $\mathfrak{S}_{n}$, which is a Weyl group of type $B_{n}$ (cf. [21]).

Lemma 2.1 For any commutative $\mathbb{Z}$-algebra $R$, there is a right $R\left[\mathfrak{S}_{2 n}\right]$-module isomorphism

$$
\mathfrak{B}_{n, R} \cong \operatorname{Ind}_{R\left[\mathbb{Z}_{22} \mathfrak{S}_{n}\right]}^{R\left[\mathfrak{S}_{2 n}\right]} 1_{R},
$$

where $1_{R}$ denotes the rank one trivial representation of $R\left[\mathbb{Z}_{2} 2 \mathfrak{S}_{n}\right]$.

Proof Let $1_{\mathfrak{B}_{n}}$ be the element in $\mathrm{Bd}_{n}$ that connects $2 i-1$ to $2 i$ for each integer $i$ with $1 \leq i \leq n$. Since $\mathfrak{S}_{2 n}$ acts transitively on the set of all the Brauer $n$-diagrams, it is easy to see that the map $\xi_{R}$ which send $1_{R}$ to $1_{\mathfrak{B}_{n}}$ extends naturally to a surjective $R\left[\mathfrak{S}_{2 n}\right]$-module homomorphism from $\operatorname{Ind}_{R\left[\mathbb{Z}_{22} \mathfrak{S}_{n}\right]}^{R\left[\mathfrak{S}_{2}\right]} 1_{R}$ onto $\mathfrak{B}_{n, R}$.

If $R$ is a field, then we can compare the dimensions of both modules. In that case, we know that the surjection $\xi_{R}$ must be an injection, and hence be an isomorphism. In general, since there are natural isomorphisms

$$
\operatorname{Ind}_{R\left[\mathbb{Z}_{2} 2 \mathfrak{S}_{n}\right]}^{R\left[\mathfrak{S}_{2 n}\right]} 1_{R} \cong R \otimes_{\mathbb{Z}} \operatorname{Ind}_{\mathbb{Z}\left[\mathbb{Z}_{2}\left(\mathfrak{S}_{n}\right]\right.}^{\mathbb{Z}\left[\mathfrak{S}_{2 n}\right]} 1_{\mathbb{Z}}, \quad \mathfrak{B}_{n, R} \cong R \otimes_{\mathbb{Z}} \mathfrak{B}_{n, \mathbb{Z}}
$$

and $\xi_{R}$ is naturally identified with $1_{R} \otimes_{\mathbb{Z}} \xi_{\mathbb{Z}}$, it suffices to show that $\xi_{\mathbb{Z}}$ is an isomorphism. Note also that the short exact sequence

$$
0 \rightarrow \operatorname{Ker} \xi_{\mathbb{Z}} \rightarrow \operatorname{Ind}_{\mathbb{Z}\left[\mathbb{Z}_{2}\left(\mathfrak{S}_{n}\right]\right.}^{\mathbb{Z}\left[\mathfrak{S}_{2 n}\right]} 1_{\mathbb{Z}} \rightarrow \mathfrak{B}_{n, \mathbb{Z}} \rightarrow 0
$$

splits as $\mathbb{Z}$-modules. It follows that $\operatorname{Ker} \xi_{R}$ is canonically isomorphic to $R \otimes_{\mathbb{Z}} \operatorname{Ker} \xi_{\mathbb{Z}}$ for any commutative $\mathbb{Z}$-algebra $R$. Let $N:=\operatorname{Ker} \xi_{\mathbb{Z}}$. It is enough to show that $N=0$. By [1, Proposition 3.8], we only need to show that $N_{(p)}=0$ for each prime number $p$. Let $k_{p}:=\mathbb{Z} /(p)$, the residue field at the prime number $p$. It is clear that $k_{p} \cong$ $\mathbb{Z}_{(p)} /(p) \mathbb{Z}_{(p)}$. Note that

$$
N_{(p)} /(p) N_{(p)} \cong k_{p} \otimes_{\mathbb{Z}_{(p)}} N_{(p)} \cong k_{p} \otimes_{\mathbb{Z}_{(p)}} \operatorname{Ker} \xi_{\mathbb{Z}_{(p)}} \cong \operatorname{Ker} \xi_{k_{p}}=0
$$

Applying Nakayama's lemma $([1,2.6])$, we conclude that $N_{(p)}=0$. This completes the proof of the lemma.

For any positive integer $k$ and any composition $\mu=\left(\mu_{1}, \cdots, \mu_{s}\right)$ of $k$, the Young diagram of $\mu$ is defined to be the set $[\mu]:=\left\{(a, b) \mid 1 \leq a \leq s, 1 \leq b \leq \mu_{a}\right\}$. The elements of $[\mu]$ are called nodes of $\mu$. A $\mu$-tableau $t$ is defined to be a bijective map 
from the Young diagram $[\mu]$ to the set $\{1,2, \cdots, k\}$. We denote by $\mathfrak{t}^{\mu}$ the $\mu$-tableau in which the numbers $1,2, \cdots, k$ appear in order along successive rows. The row stabilizer of $\mathfrak{t}^{\mu}$, denoted by $\mathfrak{S}_{\mu}$, is the standard Young subgroup of $\mathfrak{S}_{k}$ corresponding to $\mu$. For example, if $k=6, \mu=(2,3,1)$, then

$\mathfrak{t}^{\mu}={ }_{6} 45, \quad \mathfrak{S}_{\mu}=$ the subgroup of $\mathfrak{S}_{6}$ generated by $\left\{s_{1}, s_{3}, s_{4}\right\}$.

We define

$$
x_{\mu}=\sum_{w \in \mathfrak{S}_{\mu}} w, \quad y_{\mu}=\sum_{w \in \mathfrak{S}_{\mu}}(-1)^{\ell(w)} w,
$$

where $\ell(-)$ is the length function in $\mathfrak{S}_{k}$. If $\mu$ is a partition of $k$, we denote by $\mathfrak{t}_{\mu}$ the $\mu$ tableau in which the numbers $1,2, \cdots, k$ appear in order along successive columns. Let $w_{\mu} \in \mathfrak{S}_{k}$ be such that $\mathfrak{t}^{\mu} w_{\mu}=\mathfrak{t}_{\mu}$. For example, if $k=8, \mu=(3,3,1,1)$, then

$$
\mathfrak{t}^{\mu}=\begin{aligned}
& 123 \\
& 456 \\
& 7 \\
& 8
\end{aligned}, \quad \begin{array}{r}
157 \\
268 \\
3 \\
4
\end{array}, \quad \mathfrak{t}_{\mu}=(2,5,6,8,4)(3,7)
$$

For any partition $\mu$ of $2 n$, we define the associated Specht module $S^{\mu}$ to be the right ideal of the group algebra $\mathbb{Z}\left[\mathfrak{S}_{2 n}\right]$ generated by $y_{\mu^{\prime}} w_{\mu^{\prime}} x_{\mu}$. In particular, $S^{(2 n)}$ is the one-dimensional trivial representation of $\mathfrak{S}_{2 n}$, while $S^{\left(1^{2 n}\right)}$ is the one dimensional sign representation of $\mathfrak{S}_{2 n}$. By [13, Theorem 3.5] and [31, 5.3], our $S^{\mu}$ is isomorphic to the dual Specht module $\widetilde{S}^{\mu}$ introduced in [31, Section 5]. For any commutative $\mathbb{Z}$ algebra $R$, we write $S_{R}^{\mu}:=R \otimes_{\mathbb{Z}} S^{\mu}$. Then $\left\{S_{\mathbb{Q}}^{\mu} \mid \mu \vdash 2 n\right\}$ is a complete set of pairwise non-isomorphic simple $\mathbb{Q}\left[\mathfrak{S}_{2 n}\right]$-modules.

For any composition $\lambda=\left(\lambda_{1}, \cdots, \lambda_{s}\right)$ of $n$, let $2 \lambda:=\left(2 \lambda_{1}, \cdots, 2 \lambda_{s}\right)$, which is a composition of $2 n$. We use $\mathcal{P}_{n}$ to denote the set of partitions of $n$. We define $2 \mathcal{P}_{n}:=$ $\left\{2 \lambda \mid \lambda \in \mathcal{P}_{n}\right\}$.

Lemma 2.2 There is an isomorphism of right $\mathbb{Q}\left[\mathfrak{S}_{2 n}\right]$-modules:

$$
\mathfrak{B}_{n, \mathbb{Q}} \cong \bigoplus_{\lambda \in 2 \mathcal{P}_{n}} S_{\mathbb{Q}}^{\lambda}
$$

Proof This follows from Lemma 2.1 and [27, Chapter VII, (2.4)].

Let $a$ be an integer with $0 \leq a \leq n$. Let $1 \leq i_{1}, \cdots, i_{a}, j_{1}, \cdots, j_{a} \leq 2 n$ be $2 a$ distinct integers. Let

$$
I:=\{1,2, \cdots, 2 n\} \backslash\left\{i_{1}, \cdots, i_{a}, j_{1}, \cdots, j_{a}\right\} .
$$

Let $\mathfrak{S}_{I}$ be the symmetric group on the set $I$. Let $\underline{i}:=\left(i_{1}, \cdots, i_{a}\right), \underline{j}:=\left(j_{1}, \cdots, j_{a}\right)$. Let

$$
\operatorname{Bd}_{n}(\underline{i}, \underline{j}):=\left\{D \in \mathrm{Bd}_{n} \mid D \text { connects } i_{s} \text { with } j_{s} \text { for each } 1 \leq s \leq a\right\}
$$


Lemma 2.3 With the notations as above, for any $w \in \mathfrak{S}_{I}$, we have

$$
\left(\sum_{D \in \operatorname{Bd}_{n}(\underline{i}, \underline{j})} D\right) * w=\sum_{D \in \operatorname{Bd}_{n}(\underline{i}, \underline{j})} D .
$$

Proof For any $D \neq D^{\prime} \in \operatorname{Bd}_{n}(\underline{i}, \underline{j})$, it is clear that

$$
D * w \neq D^{\prime} * w \in \operatorname{Bd}_{n}(\underline{i}, \underline{j}) .
$$

Therefore, the lemma follows easily from a counting argument.

Definition 2.4 For any non-negative even integers $a, b$ with $a+b \leq 2 n$, we define

$$
\begin{aligned}
\mathrm{Bd}_{(b)}^{(a)} & :=\left\{\begin{array}{l|l}
D \in \mathrm{Bd}_{n} & \begin{array}{c}
\text { the vertex labeled by } i \text { is connected with } \\
\text { the vertex labeled by } \gamma(i) \text { whenever } \\
i \leq a \text { or } i>a+b
\end{array}
\end{array}\right\}, \\
X_{(b)}^{(a)} & :=\sum_{D \in \mathrm{Bd}_{(b)}^{(a)}} D .
\end{aligned}
$$

For any even integer $k$ with $0 \leq k \leq 2 n$, let $X_{(k)}:=X_{(k)}^{(0)}$.

Definition 2.5 Let $\lambda=\left(\lambda_{1}, \cdots, \lambda_{s}\right)$ be a composition of $2 n$ such that $\lambda_{i}$ is even for each $i$, and define

$$
X_{\lambda}:=X_{\left(\lambda_{1}\right)}^{(0)} X_{\left(\lambda_{2}\right)}^{\left(\lambda_{1}\right)} \cdots X_{\left(\lambda_{s}\right)}^{\left(\lambda_{1}+\cdots+\lambda_{s-1}\right)} \in \mathfrak{B}_{n}
$$

Note that $X_{\lambda}$ is nothing but a sum of all the Brauer $n$-diagram $D$ which satisfies the following condition: for each integer $1 \leq i \leq s$ and each integer $a$ with $\sum_{j=1}^{i-1} \lambda_{j}+$ $1 \leq a \leq \sum_{j=1}^{i} \lambda_{j}$, the vertex labeled by $a$ in $D$ can only be connected with a vertex labeled by $b$ for some integer $b$ with $\sum_{j=1}^{i-1} \lambda_{j}+1 \leq b \leq \sum_{j=1}^{i} \lambda_{j}$ and $b \neq a$.

Corollary 2.6 Let $\lambda=\left(\lambda_{1}, \cdots, \lambda_{s}\right)$ be a composition of $2 n$ such that $\lambda_{i}$ is even for each $i$. Then, for any $w \in \mathfrak{S}_{\lambda}$, we have that

$$
X_{\lambda} * w=X_{\lambda}
$$

Proof For any non-negative even integers $a, b$ with $a+b \leq 2 n$, the set $\mathrm{Bd}_{(b)}^{(a)}$ is just a special case of the set $\operatorname{Bd}_{n}(\underline{i}, j)$ we defined before. Therefore, by Lemma 2.3, for any $w \in \mathfrak{S}_{\{a+1, a+2, \cdots, a+b\}}$, we have

$$
X_{(b)}^{(a)} * w=X_{(b)}^{(a)}
$$

Now we note that the elements $X_{\left(\lambda_{1}\right)}^{(0)}, X_{\left(\lambda_{2}\right)}^{\left(\lambda_{1}\right)}, \cdots, X_{\left(\lambda_{s}\right)}^{\left(\lambda_{1}+\cdots+\lambda_{s-1}\right)}$ pairwise commute with each other. Hence the corollary follows at once. 
Let $k$ be a positive integer and $\mu$ be a composition of $k$. A $\mu$-tableau $\mathfrak{t}$ is called row standard if the numbers increase along rows. We use $\operatorname{Row} \operatorname{Std}(\mu)$ to denote the set of all the row-standard $\mu$-tableaux. Suppose $\mu$ is a partition of $k$. Then $\mathfrak{t}$ is called column standard if the numbers increase down columns, and standard if it is both row and column standard. In this case, it is clear that both $\mathfrak{t}^{\mu}$ and $\mathfrak{t}_{\mu}$ are standard $\mu$ tableaux. We use $\operatorname{Std}(\mu)$ to denote the set of all the standard $\mu$-tableaux. Let $\lambda \in 2 \mathcal{P}_{n}$. For any $\mathfrak{t} \in \operatorname{RowStd}(\lambda)$, let $d(\mathfrak{t}) \in \mathfrak{S}_{2 n}$ be such that $\mathfrak{t}^{\lambda} d(\mathfrak{t})=\mathfrak{t}$. Let $X_{\lambda, \mathfrak{t}}:=X_{\lambda} * d(\mathfrak{t})$. For any commutative $\mathbb{Z}$-algebra $R$, we define

$$
\mathcal{M}_{R}^{\lambda}:=R-\operatorname{Span}\left\{X_{v, \mathfrak{t}} \mid \mathfrak{t} \in \operatorname{Std}(v), \lambda \unlhd v \in 2 \mathcal{P}_{n}\right\},
$$

where " $\unrhd$ " is the usual dominance order, defined for example in [31]. We write $\mathcal{M}^{\lambda}=$ $\mathcal{M}_{\mathbb{Z}}^{\lambda}$. We are interested in the module $\mathcal{M}_{R}^{\lambda}$. In the remaining part of this paper, we shall see that this module is actually a right $\mathfrak{S}_{2 n}$-submodule of $\mathfrak{B}_{n, R}$, and it shares many properties with the permutation module $x_{\lambda} \mathbb{Z}\left[\mathfrak{S}_{2 n}\right]$. In particular, it also has a Specht filtration, and it is stable under base change, i.e., $R \otimes_{\mathbb{Z}} \mathcal{M}^{\lambda} \cong \mathcal{M}_{R}^{\lambda}$ for any commutative $\mathbb{Z}$-algebra $R$.

For our purpose, we need to recall some results in [31] and [28] on the Specht filtrations of permutation modules over the symmetric group $\mathfrak{S}_{2 n}$. Let $\lambda, \mu$ be two partitions of $2 n$. A $\mu$-tableau of type $\lambda$ is a map $S:[\mu] \rightarrow\{1,2, \cdots, 2 n\}$ such that each $i$ appears exactly $\lambda_{i}$ times. $\mathrm{S}$ is said to be semistandard if each row of $\mathrm{S}$ is weakly increasing and each column of $\mathrm{S}$ is strictly increasing. Let $\mathcal{T}_{0}(\mu, \lambda)$ be the set of all the semistandard $\mu$-tableaux of type $\lambda$. Then $\mathcal{T}_{0}(\mu, \lambda) \neq \emptyset$ only if $\mu \unrhd \lambda$. For each standard $\mu$-tableau $\mathfrak{s}$, let $\lambda(\mathfrak{s})$ be the tableau which is obtained from $\mathfrak{s}$ by replacing each entry $i$ in $\mathfrak{s}$ by $r$ if $i$ appears in row $r$ of $\mathfrak{t}^{\lambda}$. Then $\lambda(\mathfrak{s})$ is a $\mu$-tableau of type $\lambda$.

For each standard $\mu$-tableau $\mathfrak{t}$ and each semistandard $\mu$-tableau $S$ of type $\lambda$, we define

$$
x_{\mathrm{S}, \mathfrak{t}}:=\sum_{\mathfrak{s} \in \operatorname{Std}(\mu), \lambda(\mathfrak{s})=\mathrm{S}} d(\mathfrak{s})^{-1} x_{\mu} d(\mathfrak{t}) .
$$

Then by [31, Section 7], the set

$$
\left\{x_{\mathrm{S}, \mathfrak{t}} \mid \mathrm{S} \in \mathcal{T}_{0}(\mu, \lambda), \mathfrak{t} \in \operatorname{Std}(\mu), \lambda \unlhd \mu \vdash 2 n\right\}
$$

form a $\mathbb{Z}$-basis of $x_{\lambda} \mathbb{Z}\left[\mathfrak{S}_{2 n}\right]$. Furthermore, for any commutative $\mathbb{Z}$-algebra $R$, the canonical surjective homomorphism $R \otimes_{\mathbb{Z}} x_{\lambda} \mathbb{Z}\left[\mathfrak{S}_{2 n}\right] \rightarrow x_{\lambda} R\left[\mathfrak{S}_{2 n}\right]$ is an isomorphism.

For each partition $\mu$ of $2 n$ and for each semistandard $\mu$-tableau $S$ of type $\lambda$, according to the results in [31, Section 7] and [28], both the following $\mathbb{Z}$-submodules

$$
\begin{aligned}
& M_{\mathrm{S}}^{\lambda}:= \mathbb{Z}-\operatorname{Span}\left(\left\{x_{\mathrm{S}, \mathfrak{s}} \mid \mathfrak{s} \in \operatorname{Std}(\mu)\right\}\right. \\
&\left.\cup\left\{x_{\mathrm{T}, \mathfrak{t}} \mid \mathrm{T} \in \mathcal{T}_{0}(\nu, \lambda), \mathfrak{t} \in \operatorname{Std}(\nu), \mu \triangleleft v \vdash 2 n\right\}\right), \\
& M_{\mathrm{S}, \triangleright}^{\lambda}:=\mathbb{Z}-\operatorname{Span}\left\{x_{\mathrm{T}, \mathfrak{t}} \mid \mathrm{T} \in \mathcal{T}_{0}(\nu, \lambda), \mathfrak{t} \in \operatorname{Std}(\nu), \mu \triangleleft v \vdash 2 n\right\},
\end{aligned}
$$


are $\mathbb{Z}\left[\mathfrak{S}_{2 n}\right]$-submodules, and the quotient of $M_{\mathrm{S}}^{\lambda}$ by $M_{\mathrm{S}, \triangleright}^{\lambda}$ is canonically isomorphic to $S^{\mu}$ so that the image of the elements $x_{\mathrm{S}, \mathfrak{s}}$, where $\mathfrak{s} \in \operatorname{Std}(\mu)$, forms the standard $\mathbb{Z}$-basis of $S^{\mu}$. In other words, it gives rise to the Specht filtrations of $x_{\lambda} \mathbb{Z}\left[\mathfrak{S}_{2 n}\right]$, each semistandard $\mu$-tableau of type $\lambda$ yields a factor which is isomorphic to $S^{\mu}$ so that $x_{\lambda} \mathbb{Z}\left[\mathfrak{S}_{2 n}\right]$ has a series of factors, ordered by $\unlhd$, each isomorphic to some $S^{\mu}, \mu \unrhd \lambda$, the multiplicity of $S^{\mu}$ being the number of semistandard $\mu$-tableaux of type $\lambda$.

We write $\lambda=\left(\lambda_{1}, \lambda_{2}, \cdots\right)=\left(a_{1}^{k_{1}}, a_{2}^{k_{2}}, \cdots,\right)$, where $a_{1}>a_{2}>\cdots, k_{i} \in \mathbb{N}$ for each $i$, where $a_{i}^{k_{i}}$ means that $a_{i}$ repeats $k_{i}$ times. Let $\widetilde{\mathfrak{S}}_{\lambda}$ be the subgroup of $\mathfrak{S}_{\lambda^{\prime}}$ consisting of all the elements $w$ satisfying the following condition: for any integers $i, j$ with $\lambda_{i}=\lambda_{j}$, and any integers $a, b$ with $1 \leq a, b \leq \lambda_{i}$,

$$
\left(\mathfrak{t}_{\lambda}(i, a)\right) w=\mathfrak{t}_{\lambda}(j, a) \quad \text { if and only if } \quad\left(\mathfrak{t}_{\lambda}(i, b)\right) w=\mathfrak{t}_{\lambda}(j, b) .
$$

Let $\widetilde{D}_{\lambda}$ be a complete set of right coset representatives of $\widetilde{\mathfrak{S}}_{\lambda}$ in $\mathfrak{S}_{\lambda^{\prime}}$. By convention, we set $0 !=1$.

Lemma 2.7 For any partition $\lambda \in 2 \mathcal{P}_{n}$, let

$$
n_{\lambda}:=\prod_{i \geq 1}\left(\lambda_{i}^{\prime}-\lambda_{i+1}^{\prime}\right) !, \quad h_{\lambda}:=\sum_{w \in \widetilde{D}_{\lambda}}(-1)^{\ell(w)} w .
$$

Then $\left|\widetilde{\mathfrak{S}}_{\lambda}\right|=n_{\lambda}$, and

$$
X_{\lambda} *\left(w_{\lambda} y_{\lambda^{\prime}}\right)=n_{\lambda}\left(X_{\lambda} *\left(w_{\lambda} h_{\lambda}\right)\right)
$$

and for any commutative $\mathbb{Z}$-algebra $R, 1_{R} \otimes_{\mathbb{Z}}\left(X_{\lambda} *\left(w_{\lambda} h_{\lambda}\right)\right) \neq 0$ in $\mathfrak{B}_{n, R}$.

Proof By definition,

$$
y_{\lambda^{\prime}}=\sum_{w \in \mathfrak{S}_{\lambda^{\prime}}}(-1)^{\ell(w)} w=\left(\sum_{w \in \widetilde{\mathfrak{S}}_{\lambda}}(-1)^{\ell(w)} w\right) h_{\lambda} .
$$

Note that the condition $\lambda \in 2 \mathcal{P}_{n}$ implies that for any $w \in \widetilde{\mathfrak{S}}_{\lambda}, \ell(w)$ is an even integer. Therefore,

$$
y_{\lambda^{\prime}}=\left(\sum_{w \in \widetilde{\mathfrak{S}}_{\lambda}} w\right) h_{\lambda}
$$

Since for each $w \in \widetilde{\mathfrak{S}}_{\lambda}$,

$$
X_{\lambda} *\left(w_{\lambda} w\right)=X_{\lambda} *\left(w_{\lambda} w w_{\lambda}^{-1}\right) * w_{\lambda}=X_{\lambda} * w_{\lambda},
$$

it follows that

$$
X_{\lambda} *\left(w_{\lambda} \sum_{w \in \widetilde{\mathfrak{S}}_{\lambda}} w\right)=n_{\lambda} X_{\lambda} * w_{\lambda},
$$

from which the first statement of this lemma follows. Let $d$ be the Brauer $n$-diagram in which the vertex labeled by $\mathfrak{t}_{\lambda}(i, 2 j-1)$ is connected with the vertex labeled by 
$\mathfrak{t}_{\lambda}(i, 2 j)$ for any $1 \leq i \leq \lambda_{1}^{\prime}, 1 \leq j \leq \lambda_{i} / 2$. Then it is easy to see that $d$ appears with coefficient 1 in the expression of $X_{\lambda} *\left(w_{\lambda} h_{\lambda}\right)$ as linear combinations of basis of Brauer $n$-diagrams. It follows that for any commutative $\mathbb{Z}$-algebra $R, 1_{R} \otimes_{\mathbb{Z}}\left(X_{\lambda} *\right.$ $\left.\left(w_{\lambda} h_{\lambda}\right)\right) \neq 0$ in $\mathfrak{B}_{n, R}$, as required.

Let $\mathfrak{t}$ be a tableau with entries in $\{1,2, \cdots, k\}$. For each integer $a$ with $1 \leq a \leq k$, we define $\operatorname{res}_{\mathfrak{t}}(a)=j-i$ if $\mathfrak{t}(i, j)=a$. Following [30], we define the Jucys-Murphy operators of $\mathbb{Z}\left[\mathfrak{S}_{2 n}\right]$.

$$
\left\{\begin{array}{l}
L_{1}:=0, \\
L_{a}:=(a-1, a)+(a-2, a)+\cdots+(1, a), \quad a=2,3, \cdots, 2 n .
\end{array}\right.
$$

Then for each partition $\lambda$ of $2 n$, and each integer $1 \leq a \leq 2 n$, we have (by [13, (3.14)])

$$
\left(x_{\lambda} w_{\lambda} y_{\lambda^{\prime}}\right) L_{a}=\operatorname{res}_{\mathfrak{t}_{\lambda}}(a)\left(x_{\lambda} w_{\lambda} y_{\lambda^{\prime}}\right)
$$

For each standard $\lambda$-tableau $t$, we define

$$
\Theta_{\mathfrak{t}}:=\prod_{i=1}^{n} \prod_{\substack{\mathfrak{u} \in \operatorname{Std}(\lambda) \\ \operatorname{res}_{\mathfrak{u}}(i) \neq \operatorname{res}_{\mathfrak{t}}(i)}} \frac{L_{i}-\operatorname{res}_{\mathfrak{u}}(i)}{\operatorname{res}_{\mathfrak{t}}(i)-\operatorname{res}_{\mathfrak{u}}(i)}
$$

For each partition $\lambda \in 2 \mathcal{P}_{n}$, by Corollary 2.6 and Frobenius reciprocity, there is a surjective right $\mathbb{Z}\left[\mathfrak{S}_{2 n}\right]$-module homomorphism $\pi_{\lambda}$ from $x_{\lambda} \mathbb{Z}\left[\mathfrak{S}_{2 n}\right]$ onto $X_{\lambda} \mathbb{Z}\left[\mathfrak{S}_{2 n}\right]$ which is defined by $x_{\lambda} h \mapsto X_{\lambda} * h, \forall h \in \mathbb{Z}\left[\mathfrak{S}_{2 n}\right]$. In particular, by Lemma 2.7,

$$
\left(X_{\lambda} * w_{\lambda} h_{\lambda}\right) * L_{a}=\operatorname{res}_{\mathfrak{t}_{\lambda}}(a)\left(X_{\lambda} *\left(w_{\lambda} h_{\lambda}\right)\right) .
$$

Proposition 2.8 For any partition $\lambda \in 2 \mathcal{P}_{n}$, we have that

$$
\left[X_{\lambda} \mathbb{Q}\left[\mathfrak{S}_{2 n}\right]: S_{\mathbb{Q}}^{\lambda}\right]=1
$$

Proof By Lemma 2.2, we have

$$
\mathfrak{B}_{n, \mathbb{Q}} \cong \bigoplus_{\mu \in 2 \mathcal{P}_{n}} S_{\mathbb{Q}}^{\mu}
$$

It is well-known that each $S_{\mathbb{Q}}^{\mu}$ has a basis $\left\{v_{\mathfrak{t}}\right\}_{\mathfrak{t} \in \operatorname{Std}(\mu)}$ satisfying

$$
v_{\mathfrak{t}} L_{i}=\operatorname{res}_{\mathfrak{t}}(i) v_{\mathfrak{t}}, \quad \forall 1 \leq i \leq n
$$

Let $\lambda$ be a fixed partition in $2 \mathcal{P}_{n}$. Since $X_{\lambda} \mathbb{Q}\left[\mathfrak{S}_{2 n}\right] \subseteq \mathfrak{B}_{n}, \mathbb{Q}$, we can write

$$
X_{\lambda} *\left(w_{\lambda} h_{\lambda}\right)=\sum_{\mu \in 2 \mathcal{P}_{n}} \sum_{\mathfrak{t} \in \operatorname{Std}(\mu)} A_{\mathfrak{t}} v_{\mathfrak{t}}
$$

where $A_{\mathfrak{t}} \in \mathbb{Q}$ for each $\mathfrak{t}$. 
For each $\mu \in 2 \mathcal{P}_{n}$ and each $\mathfrak{t} \in \operatorname{Std}(\mu)$, we apply the operator $\Theta_{\mathfrak{t}}$ on both sides of the above identity and use Lemma 2.7 and the above discussion. We get that $A_{\mathfrak{t}} \neq 0$ if and only if $\mu=\lambda$ and $\mathfrak{t}=\mathfrak{t}_{\lambda}$. In other words, $X_{\lambda} *\left(w_{\lambda} h_{\lambda}\right)=A_{\mathfrak{t}_{\lambda}} v_{\mathfrak{t}_{\lambda}}$ for some $0 \neq A_{\mathfrak{t}_{\lambda}} \in \mathbb{Q}$. This implies that the projection from $X_{\lambda} \mathbb{Q}\left[\mathfrak{S}_{2 n}\right]$ to $S_{\mathbb{Q}}^{\lambda}$ is nonzero. Hence, $\left[X_{\lambda} \mathbb{Q}\left[\mathfrak{S}_{2 n}\right]: S_{\mathbb{Q}}^{\lambda}\right]=1$, as required.

For each partition $\lambda \in 2 \mathcal{P}_{n}$, by the natural surjective $\mathbb{Z}\left[\mathfrak{S}_{2 n}\right]$-module homomorphism $\pi_{\lambda}$ from $x_{\lambda} \mathbb{Z}\left[\mathfrak{S}_{2 n}\right]$ onto $X_{\lambda} \mathbb{Z}\left[\mathfrak{S}_{2 n}\right]$, we know that the elements $\pi_{\lambda}\left(x_{\mathrm{S}, \mathfrak{t}}\right)$, where $\mathrm{S} \in \mathcal{T}_{0}(\mu, \lambda), \mathfrak{t} \in \operatorname{Std}(\mu), \lambda \unlhd \mu \vdash 2 n$, span $X_{\lambda} \mathbb{Z}\left[\mathfrak{S}_{2 n}\right]$ as $\mathbb{Z}$-module.

Proposition 2.9 For any two partitions $\lambda, \mu$ of $2 n$, and for any $\mathrm{S} \in \mathcal{T}_{0}(\mu, \lambda)$, we have that $\pi_{\lambda}\left(M_{\mathrm{S}}^{\lambda}\right) \subseteq \mathcal{M}^{\lambda}$. In particular, $X_{\lambda} \mathbb{Z}\left[\mathfrak{S}_{2 n}\right] \subseteq \mathcal{M}^{\lambda}$.

Proof We first prove a weak version of the claim in this proposition. That is, for any two partitions $\lambda, \mu$ of $2 n$, and for any $\mathrm{S} \in \mathcal{T}_{0}(\mu, \lambda)$,

$$
\pi_{\lambda}\left(M_{\mathrm{S}}^{\lambda}\right) \subseteq \mathcal{M}_{\mathbb{Q}}^{\lambda}
$$

We consider the dominance order " $\unlhd$ " and use induction on $\lambda$. We start with the partition $(2 n)$, which is the unique maximal partition of $2 n$ with respect to " $\unlhd$ ". In this case, $x_{(2 n)} \mathbb{Z}\left[\mathfrak{S}_{2 n}\right]=\mathbb{Z} x_{(2 n)}$, and $X_{(2 n)} \mathbb{Z}\left[\mathfrak{S}_{2 n}\right]=\mathbb{Z} X_{(2 n)}$, it is easy to see the claim in this proposition is true for $\lambda=(2 n)$.

Now let $\lambda \triangleleft(2 n)$ be a partition of $2 n$. Assume that for any partition $v$ of $2 n$ satisfying $v \triangleright \lambda$, the claim in this proposition is true. We now prove the claim for the partition $\lambda$.

Let $\mu \unrhd \lambda$ be a partition of $2 n$ with $\mathcal{T}_{0}(\mu, \lambda) \neq \emptyset$. We consider again the dominance order " $\unlhd$ " and use induction on $\mu$. Since $\mathcal{T}_{0}((2 n), \lambda)$ contains a unique element $S_{\star}$, $\operatorname{Std}((2 n))=\left\{\mathfrak{t}^{(2 n)}\right\}$ and

$$
\pi_{\lambda}\left(x_{\mathrm{S}_{\star}, \mathfrak{t}^{(2 n)}}\right)=\pi_{\lambda}\left(x_{(2 n)}\right)=X_{(2 n)} \in \mathcal{M}^{\lambda} .
$$

So in this case the claim of this proposition is still true.

Now let $\mu \unrhd \lambda$ be a partition of $2 n$ with $\mathcal{T}_{0}(\mu, \lambda) \neq \emptyset$ and $\mu \triangleleft(2 n)$. Assume that for any partition $v$ of $2 n$ satisfying $\mathcal{T}_{0}(\nu, \lambda) \neq \emptyset$ and $\nu \triangleright \mu$, and for any $S \in \mathcal{T}_{0}(\nu, \lambda)$,

$$
\pi_{\lambda}\left(M_{\mathrm{S}}^{\lambda}\right) \subseteq \mathcal{M}_{\mathbb{Q}}^{\lambda}
$$

Let $\mathrm{S} \in \mathcal{T}_{0}(\mu, \lambda)$. The homomorphism $\pi_{\lambda}$ induces a surjective map from $M_{\mathrm{S}}^{\lambda} / M_{\mathrm{S}, \triangleright}^{\lambda}$ onto

$$
\left(\pi_{\lambda}\left(M_{\mathrm{S}}^{\lambda}\right)\right) /\left(\pi_{\lambda}\left(M_{\mathrm{S}, \triangleright}^{\lambda}\right)\right) .
$$

Hence it also induces a surjective map $\tilde{\pi}_{\lambda}$ from

$$
\left(\mathbb{Q} \otimes_{\mathbb{Z}} M_{\mathrm{S}}^{\lambda}\right) /\left(\mathbb{Q} \otimes_{\mathbb{Z}} M_{\mathrm{S}, \triangleright}^{\lambda}\right) \cong \mathbb{Q} \otimes_{\mathbb{Z}}\left(M_{\mathrm{S}}^{\lambda} / M_{\mathrm{S}, \triangleright}^{\lambda}\right) \cong S_{\mathbb{Q}}^{\mu}
$$

onto

$$
\mathbb{Q} \otimes_{\mathbb{Z}}\left(\pi_{\lambda}\left(M_{\mathrm{S}}^{\lambda}\right) / \pi_{\lambda}\left(M_{\mathrm{S}, \triangleright}^{\lambda}\right)\right)
$$


Since $S_{\mathbb{Q}}^{\mu}$ is irreducible, the above map is either a zero map or an isomorphism. If it is a zero map, then (by induction hypothesis)

$$
\pi_{\lambda}\left(M_{\mathrm{S}}^{\lambda}\right) \subseteq \pi_{\lambda}\left(M_{\mathrm{S}, \triangleright}^{\lambda}\right) \mathbb{Q} \subseteq \mathcal{M}_{\mathbb{Q}}^{\lambda}
$$

It remains to consider the case where $\tilde{\pi}_{\lambda}$ is an isomorphism. In particular,

$$
\mathbb{Q} \otimes_{\mathbb{Z}}\left(\pi_{\lambda}\left(M_{\mathrm{S}}^{\lambda}\right) / \pi_{\lambda}\left(M_{\mathrm{S}, \triangleright}^{\lambda}\right)\right) \cong S_{\mathbb{Q}}^{\mu}
$$

Applying Lemma 2.2, we know that $\mu \in 2 \mathcal{P}_{n}$.

On the other hand, the homomorphism $\pi_{\mu}$ also induces a surjective map from $x_{\mu} \mathbb{Z}\left[\mathfrak{S}_{2 n}\right] / M_{\mathrm{S}_{0}, \triangleright}^{\mu}$ onto

$$
\left(\pi_{\mu}\left(x_{\mu} \mathbb{Z}\left[\mathfrak{S}_{2 n}\right]\right)\right) /\left(\pi_{\mu}\left(M_{\mathrm{S}_{0}, \triangleright}^{\mu}\right)\right)=X_{\mu} \mathbb{Z}\left[\mathfrak{S}_{2 n}\right] /\left(\pi_{\mu}\left(M_{\mathrm{S}_{0}, \triangleright}^{\mu}\right)\right),
$$

where $\mathrm{S}_{0}$ is the unique semistandard $\mu$-tableau in $\mathcal{T}_{0}(\mu, \mu)$. Hence it also induces a surjective map $\tilde{\pi}_{\mu}$ from

$$
\left(\mathbb{Q} \otimes_{\mathbb{Z}} x_{\mu} \mathbb{Z}\left[\mathfrak{S}_{2 n}\right]\right) /\left(\mathbb{Q} \otimes_{\mathbb{Z}} M_{\mathrm{S}_{0}, \triangleright}^{\mu}\right) \cong \mathbb{Q} \otimes_{\mathbb{Z}}\left(x_{\mu} \mathbb{Z}\left[\mathfrak{S}_{2 n}\right] / M_{\mathrm{S}_{0}, \triangleright}^{\mu}\right) \cong S_{\mathbb{Q}}^{\mu}
$$

onto

$$
\mathbb{Q} \otimes_{\mathbb{Z}}\left(X_{\mu} \mathbb{Z}\left[\mathfrak{S}_{2 n}\right] / \pi_{\mu}\left(M_{\mathrm{S}_{0}, \triangleright}^{\mu}\right)\right) \cong\left(X_{\mu} \mathbb{Q}\left[\mathfrak{S}_{2 n}\right]\right) /\left(\mathbb{Q} \otimes_{\mathbb{Z}} \pi_{\mu}\left(M_{\mathrm{S}_{0}, \triangleright}^{\mu}\right)\right) .
$$

By the Specht filtration of $M_{\mathbb{Q}}^{\mu}$, we know that $S_{\mathbb{Q}}^{\mu}$ does not occur as composition factor in $\mathbb{Q} \otimes_{\mathbb{Z}} M_{\mathrm{S}_{0}, \triangleright}^{\mu}$. Hence $S_{\mathbb{Q}}^{\mu}$ does not occur as composition factor in $\mathbb{Q} \otimes_{\mathbb{Z}}$ $\pi_{\mu}\left(M_{\mathrm{S}_{0}, \triangleright}^{\mu}\right)$. By Proposition $2.8, S_{\mathbb{Q}}^{\mu}$ occurs as composition factor with multiplicity one in $X_{\mu} \mathbb{Q}\left[\mathfrak{S}_{2 n}\right]$. Therefore, $X_{\mu} \mathbb{Q}\left[\mathfrak{S}_{2 n}\right] \neq \mathbb{Q} \otimes_{\mathbb{Z}} \pi_{\mu}\left(M_{\mathrm{S}_{0}, \triangleright}^{\mu}\right)$. It follows that $\tilde{\pi}_{\mu}$ must be an isomorphism. Hence

$$
\mathbb{Q} \otimes_{\mathbb{Z}}\left(X_{\mu} \mathbb{Z}\left[\mathfrak{S}_{2 n}\right] / \pi_{\mu}\left(M_{\mathrm{S}_{0}, \triangleright}^{\mu}\right)\right) \cong S_{\mathbb{Q}}^{\mu}
$$

We write $A=\pi_{\lambda}\left(M_{\mathrm{S}}^{\lambda}\right), B=X_{\mu} \mathbb{Z}\left[\mathfrak{S}_{2 n}\right]$. Since $S_{\mathbb{Q}}^{\mu}$ appears only once in $\mathfrak{B}_{n, \mathbb{Q}}$, it follows that $S_{\mathbb{Q}}^{\mu}$ must occur as composition factor in the module

$$
\left(\mathbb{Q} \otimes_{\mathbb{Z}} A\right) \cap\left(\mathbb{Q} \otimes_{\mathbb{Z}} B\right)=\mathbb{Q} \otimes_{\mathbb{Z}}(A \cap B) .
$$

Hence $S_{\mathbb{Q}}^{\mu}$ can not occur as composition factor in the module

$$
\left(\mathbb{Q} \otimes_{\mathbb{Z}} A\right) /\left(\mathbb{Q} \otimes_{\mathbb{Z}}(A \cap B)\right) \cong \mathbb{Q} \otimes_{\mathbb{Z}}(A / A \cap B) .
$$

Therefore, the image of the canonical projection $\mathbb{Q}_{\mathbb{Z}} A \rightarrow \mathbb{Q} \otimes_{\mathbb{Z}}(A / A \cap B)$ must be contained in the image of $\mathbb{Q} \otimes_{\mathbb{Z}} \pi_{\lambda}\left(M_{\mathrm{S}, \triangleright}^{\lambda}\right)$. However, by induction hypothesis, both $\pi_{\lambda}\left(M_{\mathrm{S}, \triangleright}^{\lambda}\right)$ and $B$ are contained in the $\mathbb{Q}$-span of $\left\{X_{\alpha, \mathfrak{u}} \mid \mathfrak{u} \in \operatorname{Std}(\alpha), \lambda \unlhd \alpha \in 2 \mathcal{P}_{n}\right\}$. It follows that

$$
\pi_{\lambda}\left(M_{\mathrm{S}}^{\lambda}\right) \subseteq \mathcal{M}_{\mathbb{Q}}^{\lambda}
$$


as required.

Now we begin to prove $\pi_{\lambda}\left(M_{\mathrm{S}}^{\lambda}\right) \subseteq \mathcal{M}^{\lambda}$. Suppose that

$$
\pi_{\lambda}\left(M_{\mathrm{S}}^{\lambda}\right) \nsubseteq \mathcal{M}^{\lambda}
$$

Then (by the $\mathbb{Z}$-freeness of $\mathfrak{B}_{n}$ ) there exist an element $x \in M_{\mathrm{S}}^{\lambda}$, some integers $a, a_{\mathfrak{u}}$, and a prime divisor $p \in \mathbb{N}$ of $a$, such that

$$
a \pi_{\lambda}(x)=\sum_{\lambda \unlhd \alpha \in 2 \mathcal{P}_{n}} \sum_{\mathfrak{u} \in \operatorname{Std}(\alpha)} a_{\mathfrak{u}} X_{\alpha} * d(\mathfrak{u}),
$$

and

$$
\Sigma_{p}:=\left\{\alpha \in 2 \mathcal{P}_{n} \mid \lambda \unlhd \alpha, p \nmid a_{\mathfrak{u}}, \text { for some } \mathfrak{u} \in \operatorname{Std}(\alpha)\right\} \neq \emptyset .
$$

We take an $\alpha \in \Sigma_{p}$ such that $\alpha$ is minimal with respect to " $\unlhd$ ". Then we take an $u \in \operatorname{Std}(\alpha)$ such that $p \nmid a_{\mathfrak{u}}$ and $\ell(d(\mathfrak{u}))$ is maximal among the elements in the set $\left\{\mathfrak{u} \in \operatorname{Std}(\alpha) \mid p \nmid a_{\mathfrak{u}}\right\}$. Let $\sigma_{\mathfrak{u}}$ be the unique element in $\mathfrak{S}_{2 n}$ such that $d(\mathfrak{u}) \sigma_{\mathfrak{u}}=w_{\alpha}$ and $\ell\left(w_{\alpha}\right)=\ell(d(\mathfrak{u}))+\ell\left(\sigma_{\mathfrak{u}}\right)$. We consider the finite field $\mathbb{F}_{p}$ as a $\mathbb{Z}$-algebra. By $[12$, (4.1)], we know that for any partitions $\beta, \gamma$ of $2 n$, and element $w \in \mathfrak{S}_{2 n}$,

$$
x_{\beta} w y_{\gamma^{\prime}} \neq 0 \text { only if } \gamma \unrhd \beta \text {; while } x_{\beta} w y_{\beta^{\prime}} \neq 0 \text { only if } w \in \mathfrak{S}_{\beta} w_{\beta} \text {. }
$$

Hence by Lemma 2.7 and the homomorphism $\pi_{\lambda}$,

$$
X_{\beta} *\left(w h_{\gamma}\right) \neq 0 \text { only if } \gamma \unrhd \beta ; X_{\beta} *\left(w h_{\beta}\right) \neq 0 \text { only if } w \in \mathfrak{S}_{\beta} w_{\beta} .
$$

Using Lemma 2.7 again, we get

$$
0=1_{\mathbb{F}_{p}} \otimes_{\mathbb{Z}}\left(a \pi_{\lambda}(x) *\left(\sigma_{\mathfrak{u}} h_{\alpha}\right)\right)=1_{\mathbb{F}_{p}} \otimes_{\mathbb{Z}}\left(a_{\mathfrak{u}} X_{\alpha} *\left(w_{\alpha} h_{\alpha}\right)\right) \neq 0,
$$

which is a contradiction. This proves that $\pi_{\lambda}\left(M_{\mathrm{S}}^{\lambda}\right) \subseteq \mathcal{M}^{\lambda}$.

Corollary 2.10 For any partition $\lambda \in 2 \mathcal{P}_{n}$ and any commutative $\mathbb{Z}$-algebra $R, \mathcal{M}_{R}^{\lambda}$ is a right $\mathfrak{S}_{2 n}$-submodule of $\mathfrak{B}_{n, R}$.

Proof This follows directly from Proposition 2.9 .

Theorem 2.11 For any partition $\lambda \in 2 \mathcal{P}_{n}$ and any commutative $\mathbb{Z}$-algebra $R$, the canonical map $R \otimes_{\mathbb{Z}} \mathcal{M}^{\lambda} \rightarrow \mathcal{M}_{R}^{\lambda}$ is an isomorphism, and the set

$$
\left\{X_{v, \mathfrak{t}} \mid \mathfrak{t} \in \operatorname{Std}(v), \lambda \unlhd v \in 2 \mathcal{P}_{n}\right\}
$$

forms an $R$-basis of $\mathcal{M}_{R}^{\lambda}$. In particular, the set

$$
\left\{X_{\lambda, \mathfrak{t}} \mid \mathfrak{t} \in \operatorname{Std}(\lambda), \lambda \in 2 \mathcal{P}_{n}\right\}
$$

forms an $R$-basis of $\mathfrak{B}_{n, R}$. 
Proof We take $\lambda=\left(2^{n}\right)$, then $X_{\lambda} \mathbb{Z}\left[\mathfrak{S}_{2 n}\right]=\mathfrak{B}_{n}$. It is well-known that $\mathfrak{B}_{n, R} \cong R \otimes_{\mathbb{Z}}$ $\mathfrak{B}_{n}$ for any commutative $\mathbb{Z}$-algebra $R$. Applying Proposition 2.9 and counting the dimension, we get that for any $\mathbb{Z}$-algebra $R$ which is a field, the set

$$
\left\{X_{\lambda, \mathfrak{t}} \mid \mathfrak{t} \in \operatorname{Std}(\lambda), \lambda \in 2 \mathcal{P}_{n}\right\}
$$

must form an $R$-basis of $\mathfrak{B}_{n, R}$. Since for any commutative $\mathbb{Z}$-algebra $R$, the natural map $r \otimes_{\mathbb{Z}} d \mapsto r d, \forall r \in R, d \in \mathfrak{B}_{n}$, defines an $R$-module isomorphism $R \otimes_{\mathbb{Z}} \mathfrak{B}_{n} \cong$ $\mathfrak{B}_{n, R}$, it follows that the above set is still an $R$-basis of $\mathfrak{B}_{n, R}$ for any commutative $\mathbb{Z}$-algebra $R$.

By the $R$-linear independence of the elements in this set and Corollary 2.10, we also get that, for any partition $\lambda \in 2 \mathcal{P}_{n}$, the set

$$
\left\{X_{v, \mathfrak{t}} \mid \mathfrak{t} \in \operatorname{Std}(v), \lambda \unlhd v \in 2 \mathcal{P}_{n}\right\}
$$

must form an $R$-basis of $\mathcal{M}_{R}^{\lambda}$. Therefore, for any commutative $\mathbb{Z}$-algebra $R$, the canonical map $R \otimes_{\mathbb{Z}} \mathcal{M}^{\lambda} \rightarrow \mathcal{M}_{R}^{\lambda}$ is an isomorphism.

Remark 2.12 Note that for any partition $\lambda \in 2 \mathcal{P}_{n}, X_{\lambda} \mathbb{Z}\left[\mathfrak{S}_{2 n}\right] \subseteq \mathcal{M}^{\lambda}$. But $X_{\lambda} \mathbb{Z}\left[\mathfrak{S}_{2 n}\right]$ is not necessarily equal to $\mathcal{M}^{\lambda}$ in general. For example, one sees easily that

$$
X_{(6,2)} \notin X_{(4,4)} \mathbb{Z}\left[\mathfrak{S}_{8}\right] .
$$

In fact, if this is not the case, then we can write

$$
X_{(6,2)}+\sum_{i} a_{i} X_{(4,4)} * w_{i}=\sum_{j} b_{j} X_{(4,4)} * w_{j}^{\prime},
$$

for some positive integers $a_{i}, b_{j}$ and some elements $w_{i}, w_{j}^{\prime} \in \mathfrak{S}_{2 n}$. However, if we express both sides into linear combinations of Brauer 4-diagrams and count the number of terms, we find that this is impossible (as the equation $15+9 a=9 b$ has no solutions in $\mathbb{Z}$ ).

Theorem 2.13 For any partition $\lambda \in 2 \mathcal{P}_{n}$ and any commutative $\mathbb{Z}$-algebra $R$, we define

$$
\mathcal{M}_{R}^{\triangleright \lambda}:=R-\operatorname{Span}\left\{X_{v, \mathfrak{t}} \mid \mathfrak{t} \in \operatorname{Std}(v), \lambda \triangleleft v \in 2 \mathcal{P}_{n}\right\} .
$$

Then $\mathcal{M}_{R}^{\triangleright \lambda}$ is a right $R\left[\mathfrak{S}_{2 n}\right]$-submodule of $\mathcal{M}_{R}^{\lambda}$, and there is a $R\left[\mathfrak{S}_{2 n}\right]$-module isomorphism

$$
\mathcal{M}_{R}^{\lambda} / \mathcal{M}_{R}^{\triangleright \lambda} \cong S_{R}^{\lambda}
$$

In particular, $\mathfrak{B}_{n, R}$ has a multiplicity free Specht filtration.

Proof It suffices to consider the case where $R=\mathbb{Z}$. We first show that

$$
\mathcal{M}_{\mathbb{Q}}^{\lambda} \cong \oplus_{\lambda \unlhd \mu \in 2 \mathcal{P}_{n}} S_{\mathbb{Q}}^{\mu}, \quad \mathcal{M}_{\mathbb{Q}}^{\triangleright \lambda} \cong \oplus_{\lambda \triangleleft \mu \in 2 \mathcal{P}_{n}} S_{\mathbb{Q}}^{\mu}
$$


For each $\mu \in 2 \mathcal{P}_{n}$, we use $\rho_{\mu}^{\lambda}$ to denote the composition of the embedding $\mathcal{M}_{\mathbb{Q}}^{\lambda} \hookrightarrow$ $\mathfrak{B}_{n, \mathbb{Q}}$ and the projection $\mathfrak{B}_{n, \mathbb{Q}} \rightarrow S_{\mathbb{Q}}^{\mu}$. Suppose that $\rho_{\mu}^{\lambda} \neq 0$. Then $\rho_{\mu}^{\lambda}$ must be a surjection. We claim that $\mu \unrhd \lambda$. In fact, if $\mu \nsupseteq \lambda$, then for any $\lambda \unlhd v \in 2 \mathcal{P}_{n}, \mu \nsupseteq v$, and $x_{v} \mathbb{Z}\left[\mathfrak{S}_{2 n}\right] w_{\mu^{\prime}} x_{\mu} w_{\mu} y_{\mu^{\prime}}=0$, hence $X_{\nu, \mathfrak{t}} *\left(w_{\mu^{\prime}} x_{\mu} w_{\mu} y_{\mu^{\prime}}\right)=0$ for any $\mathfrak{t} \in \operatorname{Std}(\nu)$. It follows that $\mathcal{M}_{\mathbb{Q}}^{\lambda}\left(w_{\mu^{\prime}} x_{\mu} w_{\mu} y_{\mu^{\prime}}\right)=0$. Therefore, $S_{\mathbb{Q}}^{\mu}\left(w_{\mu^{\prime}} x_{\mu} w_{\mu} y_{\mu^{\prime}}\right)=0$. On the other hand, since $S_{\mathbb{Q}}^{\mu} \cong x_{\mu} w_{\mu^{\prime}} y_{\mu^{\prime}} \mathbb{Q}\left[\mathfrak{S}_{2 n}\right]$, and by [25, Lemma 5.7],

$$
x_{\mu} w_{\mu^{\prime}} y_{\mu^{\prime}}\left(w_{\mu^{\prime}} x_{\mu} w_{\mu} y_{\mu^{\prime}}\right)=\left(\prod_{(i, j) \in[\mu]} h_{i, j}^{\mu}\right) x_{\mu} w_{\mu^{\prime}} y_{\mu^{\prime}} \neq 0
$$

where $h_{i, j}^{\mu}$ is the $(i, j)$-hook length in $[\mu]$, we get a contradiction. Therefore, $\rho_{\mu}^{\lambda} \neq$ 0 must imply that $\mu \unrhd \lambda$. Now counting the dimensions, we deduce that $\mathcal{M}_{\mathbb{Q}}^{\lambda} \cong$ $\oplus_{\lambda \unlhd \mu \in 2 \mathcal{P}_{n}} S_{\mathbb{Q}}^{\mu}$. In a similar way, we can prove that $\mathcal{M}_{\mathbb{Q}}^{\triangleright \lambda} \cong \oplus_{\lambda \triangleleft \mu \in 2 \mathcal{P}_{n}} S_{\mathbb{Q}}^{\mu}$. It follows that $\mathcal{M}_{\mathbb{Q}}^{\lambda} / \mathcal{M}_{\mathbb{Q}}^{\triangleright \lambda} \cong S_{\mathbb{Q}}^{\lambda}$.

We now consider the natural map from $x_{\lambda} \mathbb{Z}\left[\mathfrak{S}_{2 n}\right]$ onto $\mathcal{M}^{\lambda} / \mathcal{M}^{\triangleright \lambda} \cong S^{\lambda}$. Since $\mathbb{Q} \otimes_{\mathbb{Z}} M_{\mathrm{S}_{0}, \triangleright}^{\lambda}$ does not contain $S_{\mathbb{Q}}^{\lambda}$ as a composition factor, it follows that (by Proposition 2.9) the image of $M_{\mathrm{S}_{0}, \triangleright}^{\lambda}$ must be contained in $\mathcal{M}^{\triangleright \lambda}$. Therefore we get a surjective map from $S^{\lambda}$ onto $\mathcal{M}^{\lambda} / \mathcal{M}^{\triangleright \lambda} \cong S^{\lambda}$. This map sends the standard basis of $S^{\lambda}$ to the canonical basis of $\mathcal{M}^{\lambda} / \mathcal{M}^{\triangleright \lambda}$. So it must be injective as well, as required.

\section{The $n$-tensor space $V^{\otimes n}$}

In this section, we shall use the results obtained in Section 2 and in [11] to give an explicit and characteristic-free description of the annihilator of the $n$-tensor space $V^{\otimes n}$ in the Brauer algebra $\mathfrak{B}_{n}(-2 m)$.

Let $K$ be an arbitrary infinite field. Let $m, n \in \mathbb{N}$. Let $V$ be a $2 m$-dimensional symplectic $K$-vector space. Let $S p(V)$ be the corresponding symplectic group, acting naturally on $V$, and hence on the $n$-tensor space $V^{\otimes n}$ from the left-hand side. As we mentioned in the introduction, this left action on $V^{\otimes n}$ is centralized by the specialized Brauer algebra $\mathfrak{B}_{n}(-2 m)_{K}:=K \otimes_{\mathbb{Z}} \mathfrak{B}_{n}(-2 m)$, where $K$ is regarded as a $\mathbb{Z}$-algebra by sending each integer $a$ to $a \cdot 1_{K}$. The Brauer algebra $\mathfrak{B}_{n}(-2 m)_{K}$ acts on the $n$-tensor space $V^{\otimes n}$ from the right-hand side. Let $\varphi$ be the natural $K$-algebra homomorphism

$$
\varphi:\left(\mathfrak{B}_{n}(-2 m)_{K}\right)^{o p} \rightarrow \operatorname{End}_{K}\left(V^{\otimes n}\right) .
$$

By the discussion in [11, Section 3], $V^{\otimes n}$ is a tilting module over $K S p(V)$. By $[14,(4.4)]$, the dimension of $\operatorname{End}_{K S p(V)}\left(V^{\otimes n}\right)$ is independent of the choice of the infinite field $K$. Therefore, the dimension of $\operatorname{Ker} \varphi:=\left\{y \in \mathfrak{B}_{n}(-2 m)_{K} \mid \varphi(y)=\right.$ $0\}$ is also independent of the choice of the infinite field $K$. That is, the dimension of the annihilator of the $n$-tensor space $V^{\otimes n}$ in the Brauer algebra $\mathfrak{B}_{n}(-2 m)_{K}$ is independent of the choice of the infinite field $K$. 
Lemma 3.1 With the notations as above, we have that

$$
\operatorname{dim}(\operatorname{Ker} \varphi)=\sum_{\substack{\lambda \in 2 \mathcal{P}_{n} \\ \lambda_{1}>2 m}} \operatorname{dim} S^{\lambda}
$$

Proof By Lemma 2.2 and Lemma 1.1, it suffices to consider the case where $K=\mathbb{C}$ and to show that

$$
\operatorname{dim} \operatorname{End}_{\mathbb{C S p _ { 2 m } ( V )}}\left(V^{\otimes n}\right)=\sum_{\substack{\lambda \in 2 \mathcal{P}_{n} \\ \lambda_{1} \leq 2 m}} \operatorname{dim} S^{\lambda} .
$$

Note that $\operatorname{dim} S^{\lambda}=\operatorname{dim} S^{\lambda^{\prime}}$, and

$$
\operatorname{End}_{\mathbb{C S} p_{2 m}(V)}\left(V^{\otimes n}\right) \cong\left(\left(V^{\otimes n}\right) \otimes\left(V^{\otimes n}\right)^{*}\right)^{S p(V)} \cong\left(V^{\otimes 2 n}\right)^{S p(V)} .
$$

Therefore, it suffices to show that

$$
\operatorname{dim}\left(V^{\otimes 2 n}\right)^{S p(V)}=\sum_{\substack{\lambda \in 2 \mathcal{P}_{n} \\ \lambda_{1} \leq 2 m}} \operatorname{dim} S^{\lambda^{\prime}}
$$

By the well-known Schur-Weyl duality between the general linear group $G L(V)$ and the symmetric group $\mathfrak{S}_{2 n}$ on the tensor space $V^{\otimes 2 n}$, we know that there is a $\left(G L(V), \mathfrak{S}_{2 n}\right)$-bimodules decomposition

$$
V^{\otimes 2 n} \cong \bigoplus_{\substack{\lambda \vdash 2 n \\ \ell(\lambda) \leq 2 m}} \widetilde{\Delta}_{\lambda} \otimes S^{\lambda}
$$

where $\widetilde{\Delta}_{\lambda}$ denotes the irreducible Weyl module with highest weight $\lambda$ over GL( $\left.V\right)$. Here we identify $\lambda$ with $\lambda_{1} \varepsilon_{1}+\cdots+\lambda_{2 m} \varepsilon_{2 m}, \varepsilon_{i}$ is the weight of $G L(V)$ defined by

$$
\varepsilon_{i}\left(\operatorname{diag}\left\{t_{1}, t_{2}, \cdots, t_{2 m}\right\}\right)=t_{i}, \forall t_{1}, \cdots, t_{2 m} \in K^{\times},
$$

for $i=1,2, \cdots, 2 m$. It follows that

$$
\operatorname{dim}\left(V^{\otimes 2 n}\right)^{S p(V)}=\sum_{\substack{\lambda \vdash 2 n \\ \ell(\lambda) \leq 2 m}} \operatorname{dim}\left(\left(\widetilde{\Delta}_{\lambda} \downarrow S p(V)\right)^{S p(V)}\right) \operatorname{dim}\left(S^{\lambda}\right) .
$$

By the branching law (see [26, Proposition 2.5.1]) from $G L(V)$ to $S p(V)$, we know that

$$
\operatorname{dim}\left(\left(\widetilde{\Delta}_{\lambda} \downarrow_{S p(V)}\right)^{S p(V)}\right)=1
$$

if $\lambda^{\prime} \in 2 \mathcal{P}_{n}$; and 0 otherwise. This proves that

$$
\operatorname{dim}\left(V^{\otimes 2 n}\right)^{S p(V)}=\sum_{\substack{\lambda \in 2 \mathcal{P}_{n} \\ \lambda_{1} \leq 2 m}} \operatorname{dim} S^{\lambda^{\prime}}
$$


as required.

Let $a, b$ be two integers such that $0 \leq a, b \leq n$ and $a+b$ is even. Let

$$
I_{a}^{\text {odd }}:=\{1,3,5, \cdots, 2 a-1\}, \quad I_{b}^{\text {even }}:=\{2,4,6, \cdots, 2 b\} .
$$

If $a \geq b$, we define $\operatorname{Bd}_{n}(a, b)$ to be the set of all Brauer $n$-diagrams $D$ such that:

- for each integer $s$ with $a+1 \leq s \leq n, D$ connects the vertex labeled by $2 s-1$ with the vertex labeled by $2 s$;

- for each integer $s$ with $1 \leq s \leq(a-b) / 2, D$ connects the vertex labeled by $2 b+$ $4 s-2$ with the vertex labeled by $2 b+4 s$.

If $b \geq a$, we define $\operatorname{Bd}_{n}(a, b)$ to be the set of all Brauer $n$-diagrams $D$ such that:

- for each integer $b+1 \leq s \leq n, D$ connects the vertex labeled by $2 s-1$ with the vertex labeled by $2 s$;

- for each integer $1 \leq s \leq(b-a) / 2, d$ connects the vertex labeled by $2 a+4 s-3$ with the vertex labeled by $2 a+4 s-1$.

For example, let $D_{1}$ be the following Brauer 7-diagram

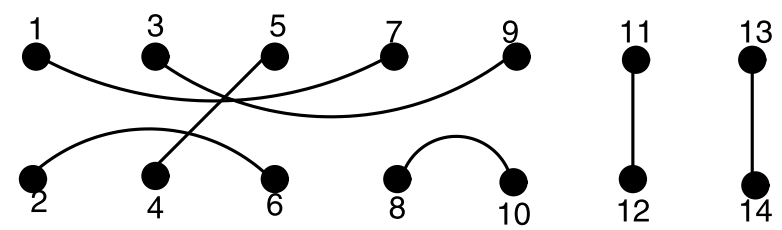

Then $D_{1} \in \operatorname{Bd}_{7}(5,3)$. Let $D_{2}$ be the following Brauer 7-diagram

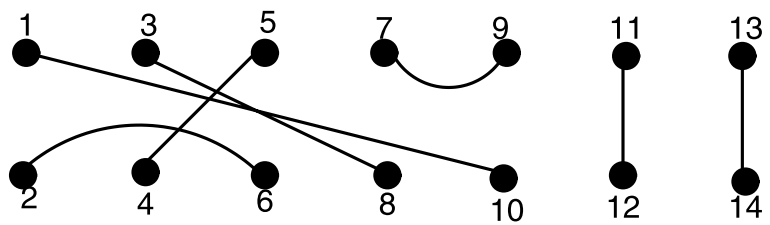

Then $D_{2} \in \operatorname{Bd}_{7}(3,5)$.

Lemma 3.2 Let $a, b$ be two integers such that $0 \leq a, b \leq n$ and $a+b$ is even. Suppose that $a+b \geq 2 m+2$, then

$$
\sum_{D \in \operatorname{Bd}_{n}(a, b)} D \in \operatorname{Ker} \varphi .
$$

The proof of Lemma 3.2 is somewhat complicated and will be given in Section 4. Given any two subsets $A^{(1)} \subseteq\{1,3, \cdots, 2 n-1\}, A^{(2)} \subseteq\{2,4, \cdots, 2 n\}$ with $\left|A^{(1)}\right|+\left|A^{(2)}\right|$ is even, we set $2 n_{0}=\left|A^{(1)}\right|+\left|A^{(2)}\right|$, and

$$
\left\{a_{1}, a_{2}, \cdots, a_{2 n-2 n_{0}}\right\}:=\{1,2, \cdots, 2 n\} \backslash\left(A^{(1)} \cup A^{(2)}\right) .
$$

Let $\left(i_{1}, j_{1}, i_{2}, j_{2}, \cdots, i_{n-n_{0}}, j_{n-n_{0}}\right)$ be a fixed permutation of

$$
\left\{a_{1}, a_{2}, \cdots, a_{2 n-2 n_{0}}\right\} \text {. }
$$


Let

$$
\mathbf{i}:=\left(i_{1}, i_{2}, \cdots, i_{n-n_{0}}\right), \quad \mathbf{j}:=\left(j_{1}, j_{2}, \cdots, j_{n-n_{0}}\right) .
$$

We define $\mathrm{Bd}_{n}^{\mathbf{i}, \mathbf{j}}\left(A^{(1)}, A^{(2)}\right)$ to be the set of all Brauer $n$-diagrams $D$ such that: for each integer $s$ with $1 \leq s \leq n-n_{0}, D$ connects the vertex labeled by $i_{s}$ with the vertex labeled by $j_{s}$. Note that the set $\operatorname{Bd}_{n}(a, b)$ we defined before is a special case of the set $\mathrm{Bd}_{n}^{\mathbf{i}, \mathbf{j}}\left(A^{(1)}, A^{(2)}\right)$ we defined here.

Corollary 3.3 With the notations as above and suppose that $2 n_{0}=\left|A^{(1)}\right|+\left|A^{(2)}\right| \geq$ $2 m+2$, then we have

$$
\sum_{D \in \operatorname{Bd}_{n}^{\mathbf{i}, \mathbf{j}}\left(A^{(1)}, A^{(2)}\right)} D \in \operatorname{Ker} \varphi
$$

Proof Let $n_{1}=\left|A^{(1)}\right|, n_{2}=\left|A^{(2)}\right|$. If $n_{1} \geq n_{2}$, then for any Brauer diagram $D \in$ $\operatorname{Bd}_{n}^{\mathbf{i}, \mathbf{j}}\left(A^{(1)}, A^{(2)}\right)$, there exist at least $\frac{n_{1}-n_{2}}{2}$ bottom horizontal edges between the vertices labeled by the integers in the following set

$$
\{2,4,6, \cdots, 2 n\} \backslash A^{(2)} \text {. }
$$

As a result, we deduce that there exist elements $\sigma_{A^{(1)}} \in \mathfrak{S}_{(1,3, \cdots, 2 n-1)}, \sigma_{A^{(2)}} \in$ $\mathfrak{S}_{(2,4, \cdots, 2 n)}$ and a Brauer diagram $D_{1}$, such that

(1) for any integer $a$ with $1 \leq a \leq n_{1}, D_{1}$ connects the vertex labeled by $2 a-1$ with the vertex labeled by $2 a$.

(2)

$$
\sigma_{A^{(1)}}\left(\sum_{D \in \mathrm{Bd}_{n}^{\mathbf{i}, j}\left(A^{(1)}, A^{(2)}\right)} D\right) \sigma_{A^{(2)}}=D_{1}\left(\sum_{D \in \operatorname{Bd}_{n}\left(\left|A^{(1)}\right|,\left|A^{(2)}\right|\right)} D\right) .
$$

In this case, since both $\varphi\left(\sigma_{A^{(1)}}\right)$ and $\varphi\left(\sigma_{A^{(2)}}\right)$ are invertible, it follows directly from Lemma 3.2 that $\sum_{D \in \mathrm{Bd}_{n}^{\mathrm{i}, \mathrm{j}}\left(A^{(1)}, A^{(2)}\right)} D \in \operatorname{Ker} \varphi$.

If $n_{1} \leq n_{2}$, then for any Brauer diagram $D \in \mathrm{Bd}_{n}^{\mathbf{i}, \mathbf{j}}\left(A^{(1)}, A^{(2)}\right)$, there exist at least $\frac{n_{2}-n_{1}}{2}$ top horizontal edges between the vertices labeled by the integers in the following set

$$
\{1,3,5, \cdots, 2 n-1\} \backslash A^{(1)} .
$$

As a result, we deduce that there exist elements $\sigma_{A^{(1)}} \in \mathfrak{S}_{(1,3, \cdots, 2 n-1)}, \sigma_{A^{(2)}} \in$ $\mathfrak{S}_{(2,4, \cdots, 2 n)}$ and a Brauer diagram $D_{2}$, such that

(3) for any integer $a$ with $1 \leq a \leq n_{2}, D_{2}$ connects the vertex labeled by $2 a-1$ with the vertex labeled by $2 a$.

(4)

$$
\sigma_{A^{(1)}}\left(\sum_{D \in \mathrm{Bd}_{n}^{\mathbf{i}, j}\left(A^{(1)}, A^{(2)}\right)} D\right) \sigma_{A^{(2)}}=D_{2}\left(\sum_{D \in \mathrm{Bd}_{n}\left(\left|A^{(1)}\right|,\left|A^{(2)}\right|\right)} D\right) .
$$


By the same argument as before, we deduce that $\sum_{D \in \mathrm{Bd}_{n}^{\mathbf{i}, \mathbf{j}}\left(A^{(1)}, A^{(2)}\right)} D \in \operatorname{Ker} \varphi$ in this case. This completes the proof of the corollary.

The following is the main result of this section, which gives an explicit and characteristic-free description of the annihilator of the $n$-tensor space $V^{\otimes n}$ in the Brauer algebra $\mathfrak{B}_{n}(-2 m)$.

Theorem 3.4 With the notations as in Lemma 1.1 and Lemma 3.1, we have that

$$
\operatorname{Ker} \varphi=\mathcal{M}_{K}^{\left(2 m+2,2^{n-m-1}\right)},
$$

where $\left(2 m+2,2^{n-m-1}\right):=(2 m+2, \underbrace{2, \cdots, 2}_{n-m-1 \text { copies }}), \mathcal{M}_{K}^{\left(2 m+2,2^{n-m-1}\right)}$ is the right $K\left[\mathfrak{S}_{2 n}\right]$-module associated to $\left(2 m+2,2^{n-m-1}\right)$ as defined in Section 2. In particular, $\operatorname{Ker} \varphi$ is a $\mathfrak{S}_{2 n}$-submodule.

Proof It is easy to see that for any partition $\mu \in 2 \mathcal{P}_{n}, \mu \unrhd\left(2 m+2,2^{n-m-1}\right)$ if and only if $\mu_{1}>2 m$. Therefore,

$$
\operatorname{dim} \mathcal{M}_{K}^{\left(2 m+2,2^{n-m-1}\right)}=\sum_{\substack{\lambda \in 2 \mathcal{P}_{n} \\ \lambda_{1}>2 m}} \operatorname{dim} S^{\lambda}
$$

Applying Lemma 1.1 and Lemma 3.1, we see that to prove this theorem, it suffices to show that $\mathcal{M}_{K}^{\left(2 m+2,2^{n-m-1}\right)} \subseteq \operatorname{Ker} \varphi$. Equivalently, it suffices to show that for any partition $\lambda=\left(\lambda_{1}, \cdots, \lambda_{s}\right) \in 2 \overline{\mathcal{P}}_{n}$ satisfying $\lambda_{1}>2 m$, and any $w \in \mathfrak{S}_{2 n}, \varphi\left(X_{\lambda} * w\right)=0$.

By the definition of the element $X_{\lambda}$, the action "*" and the multiplication rule of Brauer diagrams, we deduce that

$$
X_{\lambda} * w=\sum_{\mathbf{i}, \mathbf{j}} \sum_{D \in \mathrm{Bd}_{n}^{\mathbf{i}, \mathbf{j}}\left(A^{(1)}, A^{(2)}\right)} D
$$

where

$$
\begin{aligned}
& A^{(1)}:=\left\{(i) w \mid i=1,2,3, \cdots, \lambda_{1}\right\} \cap\{1,3,5, \cdots, 2 n-1\}, \\
& A^{(2)}:=\left\{(i) w \mid i=1,2,3, \cdots, \lambda_{1}\right\} \cap\{2,4,6, \cdots, 2 n\},
\end{aligned}
$$

and $\left|A^{(1)}\right|+\left|A^{(2)}\right|=2 n_{0}=\lambda_{1}$, and $\mathbf{i}:=\left(i_{1}, i_{2}, \cdots, i_{n-n_{0}}\right), \mathbf{j}:=\left(j_{1}, j_{2}, \cdots, j_{n-n_{0}}\right)$ such that $\left(i_{1}, j_{1}, i_{2}, j_{2}, \cdots, i_{n-n_{0}}, j_{n-n_{0}}\right)$ is a permutation of the integers in $\{1,2, \cdots$, $2 n\} \backslash\left(A^{(1)} \cup A^{(2)}\right)$. We now apply Corollary 3.3. It follows immediately that $\varphi\left(X_{\lambda} * w\right)=0$ as required. This completes the proof of the theorem.

Let $V_{\mathbb{Z}}$ be a free $\mathbb{Z}$-module with basis $\left\{v_{1}, v_{2}, \cdots, v_{2 m}\right\}$. For any commutative $\mathbb{Z}$ algebra $R$, we define $V_{R}:=R \otimes_{\mathbb{Z}} V_{\mathbb{Z}}$. We have an action of the algebra $\mathfrak{B}_{n}(-2 m)$ on $V_{\mathbb{Z}}^{\otimes n}$ which is obtained by restricting the earlier action, and hence an action of 
$\mathfrak{B}_{n}(-2 m)_{R}$ on $V_{R}^{\otimes n}$. Let $S_{R}^{s y}(m, n)$ (see [11, Section 2] and [32]) be the symplectic Schur algebra over $R$. If $R$ is a field, then $S_{R}^{s y}(m, n)$ is a quasi-hereditary algebra over $R$, and $V_{R}^{\otimes n}$ is a tilting module over $S_{R}^{s y}(m, n)$. Applying [14, (4.4)], we know that, for any commutative $\mathbb{Z}$-algebra $R$, there is a canonical isomorphism

$$
R \otimes_{\mathbb{Z}} \operatorname{End}_{S_{\mathbb{Z}}^{s y}(m, n)}\left(V_{\mathbb{Z}}^{\otimes n}\right) \cong \operatorname{End}_{S_{R}^{s y}(m, n)}\left(V_{R}^{\otimes n}\right) .
$$

Note that $\varphi\left(\mathfrak{B}_{n}(-2 m)_{\mathbb{C}}\right)=\operatorname{End}_{S_{\mathbb{C}}^{s y}(m, n)}\left(V_{\mathbb{C}}^{\otimes n}\right)$. By restriction, we get that

$$
\varphi\left(\mathfrak{B}_{n}(-2 m)_{\mathbb{Z}}\right) \subseteq \operatorname{End}_{S_{\mathbb{Z}}^{s y}(m, n)}\left(V_{\mathbb{Z}}^{\otimes n}\right)
$$

Hence for any commutative $\mathbb{Z}$-algebra $R$,

$$
\varphi\left(\mathfrak{B}_{n}(-2 m)_{R}\right) \subseteq \operatorname{End}_{S_{R}^{s y}(m, n)}\left(V_{R}^{\otimes n}\right)
$$

By the main result in [11], we know that the above inclusion " $\subseteq$ " can be replaced by "=" when $R=K$ is an infinite field. Counting dimensions, we see that the above inclusion " $\subseteq$ " can be replaced by " $=$ " when $R=K$ is an arbitrary field.

Corollary 3.5 For any commutative $\mathbb{Z}$-algebra $R$,

$$
\varphi\left(\mathfrak{B}_{n}(-2 m)_{R}\right)=\operatorname{End}_{S_{R}^{s y}(m, n)}\left(V_{R}^{\otimes n}\right)
$$

In particular, Theorem 3.4 is always true if we replace the infinite field $K$ by any commutative $\mathbb{Z}$-algebra $R$.

Proof By (3.1), it suffices to show that

$$
\varphi\left(\mathfrak{B}_{n}(-2 m)_{\mathbb{Z}}\right)=\operatorname{End}_{S_{\mathbb{Z}}^{s y}(m, n)}\left(V_{\mathbb{Z}}^{\otimes n}\right)
$$

Let $N:=\left(\operatorname{End}_{S_{\mathbb{Z}}^{s y}(m, n)}\left(V_{\mathbb{Z}}^{\otimes n}\right)\right) / \varphi\left(\mathfrak{B}_{n}(-2 m)_{\mathbb{Z}}\right)$. It is enough to show that $N=0$. By [1, Proposition 3.8], it suffices to show that $N_{(p)}=0$ for each prime number $p$. Let $k_{p}:=\mathbb{Z} /(p)$, the residue field at the prime number $p$. It is clear that $k_{p} \cong \mathbb{Z}_{(p)} /(p) \mathbb{Z}_{(p)}$. Note that

$$
\begin{aligned}
N_{(p)} /(p) N_{(p)} & \cong k_{p} \otimes_{\mathbb{Z}_{(p)}} N_{(p)} \cong k_{p} \otimes_{\mathbb{Z}} N \\
& \cong\left(\operatorname{End}_{S_{k_{p}}^{s y}(m, n)}\left(V_{k_{p}}^{\otimes n}\right)\right) / \varphi\left(\mathfrak{B}_{n}(-2 m)_{k_{p}}\right)=0 .
\end{aligned}
$$

Applying Nakayama's lemma $([1,2.6])$, we conclude that $N_{(p)}=0$. This completes the proof of the corollary. 


\section{Proof of Lemma 3.2}

We shall first fix some notations and conventions. Since the element $\sum_{D \in \mathrm{Bd}_{n}(a, b)} D$ in Lemma 3.2 actually lies in $\mathfrak{B}_{n}$, we can choose to work inside the Brauer algebra $\mathfrak{B}_{n}(-2 m)_{\mathbb{C}}$ in this section. Furthermore, throughout this section, we shall recover our original way of labeling of vertices in each Brauer $n$-diagram. That is, the vertices in each row of a Brauer $n$-diagram will be labeled by the indices $1,2, \cdots, n$ from left to right. This way of labeling is more convenient when we need to express each Brauer diagram in terms of the standard generators $s_{i}, e_{i}$ for $1 \leq i \leq n-1$ and to consider the action of Brauer diagrams on the $n$-tensor space $V^{\otimes n}$.

Let $f$ be an integer with $0 \leq f \leq[n / 2]$, where $[n / 2]$ is the largest non-negative integer not bigger than $n / 2$. Define

$$
\mathfrak{D}_{f}:=\left\{\begin{array}{l|l}
d \in \mathfrak{S}_{n} & \begin{array}{c}
(2 j-1) d<(2 j) d \text { for } 1 \leq j \leq f, \\
1 d<3 d<\cdots<(2 f-1) d \\
(2 f+1) d<(2 f+2) d<\cdots<n d
\end{array}
\end{array}\right\} .
$$

For each partition $\lambda$ of $n-2 f$, we denote by $\operatorname{Std}_{2 f}(\lambda)$ the set of all the standard $\lambda$-tableaux with entries in $\{2 f+1, \cdots, n\}$. The initial tableau $\mathfrak{t}^{\lambda}$ in this case has the numbers $2 f+1, \cdots, n$ in order along successive rows. Again, for each $\mathfrak{t} \in \operatorname{Std}_{2 f}(\lambda)$, let $d(\mathfrak{t})$ be the unique element in $\mathfrak{S}_{\{2 f+1, \cdots, n\}} \subseteq \mathfrak{S}_{n}$ with $\mathfrak{t}^{\lambda} d(\mathfrak{t})=\mathfrak{t}$. Let $\sigma \in \mathfrak{S}_{\{2 f+1, \cdots, n\}}$ and $d_{1}, d_{2} \in \mathfrak{D}_{f}$. Then $d_{1}^{-1} e_{1} e_{3} \cdots e_{2 f-1} \sigma d_{2}$ corresponds to the Brauer $n$-diagram where the top horizontal edges connect $(2 i-1) d_{1}$ and $(2 i) d_{1}$, the bottom horizontal edges connect $(2 i-1) d_{2}$ and $(2 i) d_{2}$, for $i=1,2, \cdots, f$, and the vertical edges connect $(j) d_{1}$ with $(j) \sigma d_{2}$ for $j=2 f+1,2 f+2, \cdots, n$.

Lemma 4.1 ([11, Corollary 3.3]) With the above notations, the set

$$
\left\{d_{1}^{-1} e_{1} e_{3} \cdots e_{2 f-1} \sigma d_{2} \mid 0 \leq f \leq[n / 2], \sigma \in \mathfrak{S}_{\{2 f+1, \cdots, n\}}, d_{1}, d_{2} \in \mathfrak{D}_{f}\right\}
$$

is a basis of the Brauer algebra $B_{n}(x)_{R}$, which coincides with the natural basis given by Brauer $n$-diagrams.

Given an element $d_{1}^{-1} e_{1} e_{3} \cdots e_{2 f-1} \sigma d_{2}$ as above, let $D$ be its representing Brauer $n$-diagram. Let $v_{\underline{i}}:=v_{i_{1}} \otimes v_{i_{2}} \otimes \cdots \otimes v_{i_{n}}$ be a simple $n$-tensor in $V^{\otimes n}$.

Lemma 4.2 With the notations as above, we have that

$$
\left.v_{\underline{i}} D=(-1)^{\ell\left(d_{1}^{-1} \sigma d_{2}\right)}\left(v_{\underline{i}} \circ D\right)\right),
$$

where $v_{\underline{i}} \circ D$ can be described as follows:

(1) If $(j) d_{1}^{-1} \sigma d_{2}=(k)$ for $j \in\left\{(2 f+1) d_{1},(2 f+2) d_{1}, \cdots,(n) d_{1}\right\}$, then the $k t h$ position of $v_{\underline{i}} \circ D$ is $v_{i_{j}}$;

(2) For each $1 \leq j \leq f$, the $\left((2 j-1) d_{2},(2 j) d_{2}\right)$ th position of $v_{\underline{i}} \circ D$ is the following sum:

$$
\epsilon_{i_{(2 j-1) d_{1}}, i_{(2 j) d_{1}}} \sum_{k=1}^{m}\left(v_{k^{\prime}} \otimes v_{k}-v_{k} \otimes v_{k^{\prime}}\right)
$$


Remark 4.3 Intuitively, the action of the Brauer $n$-diagram $D$ on $v_{\underline{i}}$ can be thought as follows. Let $\left(a_{1}, b_{1}\right), \cdots,\left(a_{f}, b_{f}\right)$ be the set of all the horizontal edges in the top row of $D$, where $a_{s}<b_{s}$ for each $s$ and $a_{1}<a_{2}<\cdots<a_{f}$. Let $\left(c_{1}, d_{1}\right), \cdots,\left(c_{f}, d_{f}\right)$ be the set of all the horizontal edges in the bottom row of $D$, where $c_{s}<d_{s}$ for each $s$ and $c_{1}<c_{2}<\cdots<c_{f}$. Then for each $1 \leq j \leq f$, the $\left(c_{j}, d_{j}\right)$ th position of $v_{\underline{i}} \circ D$ is the following sum:

$$
\epsilon_{i_{a_{j}}, i_{b_{j}}} \sum_{k=1}^{m}\left(v_{k^{\prime}} \otimes v_{k}-v_{k} \otimes v_{k^{\prime}}\right) .
$$

We list those vertices in the top row of $D$ which are not connected with horizontal edges from left to right as $i_{k_{2 f+1}}, i_{k_{2 f+2}}, \cdots, i_{k_{n}}$. Then, for each integer $j$ with $2 f+$ $1 \leq j \leq n$, the $\left(j \sigma d_{2}\right)$ th position of $v_{\underline{i}} \circ D$ is $v_{i_{k}}$.

Given an arbitrary element $d_{2} \in \mathfrak{D}_{f}$, we define

$$
\operatorname{Bd}^{(f)}\left(n ; d_{2}\right):=\left\{d_{1}^{-1} e_{1} e_{3} \cdots e_{2 f-1} \sigma d_{2} \mid d_{1} \in \mathfrak{D}_{f}, \sigma \in \mathfrak{S}_{\{2 f+1,2 f+2, \cdots, n\}}\right\} .
$$

Note that $\mathrm{Bd}^{(f)}\left(n ; d_{2}\right)$ consists of all the Brauer $n$-diagrams whose bottom horizontal edges are

$$
\left((1) d_{2},(2) d_{2}\right),\left((3) d_{2},(4) d_{2}\right), \cdots,\left((2 f-1) d_{2},(2 f) d_{2}\right) \text {. }
$$

Lemma 4.4 Let $f$ be an integer with $0 \leq f \leq[n / 2]$. Let $d_{2} \in \mathfrak{D}_{f}$. Then for any $\sigma \in \mathfrak{S}_{n}$,

$$
\sigma\left(\sum_{D \in \mathrm{Bd}^{(f)}\left(n ; d_{2}\right)} D\right)=\sum_{D \in \mathrm{Bd}^{(f)}\left(n ; d_{2}\right)} D
$$

Proof It suffices to show that for each integer $1 \leq i<n$,

$$
s_{i}\left(\sum_{D \in \mathrm{Bd}^{(f)}\left(n ; d_{2}\right)} D\right)=\sum_{D \in \mathrm{Bd}^{(f)}\left(n ; d_{2}\right)} D .
$$

In fact, for $D, D^{\prime} \in \mathrm{Bd}^{(f)}\left(n ; d_{2}\right)$ with $D \neq D^{\prime}$, it is clear that $s_{i} D \neq s_{i} D^{\prime}$, and both $s_{i} D$ and $s_{i} D^{\prime}$ are still lie in $\mathrm{Bd}^{(f)}\left(n ; d_{2}\right)$. Now counting the number of Brauer $n$ diagrams occurring in both sides, we prove (4.1) and hence also prove the lemma.

Similarly, given an arbitrary element $d_{1} \in \mathfrak{D}_{f}$, we define

$$
\operatorname{Bd}^{(f)}\left(d_{1} ; n\right):=\left\{d_{1}^{-1} e_{1} e_{3} \cdots e_{2 f-1} \sigma d_{2} \mid d_{2} \in \mathfrak{D}_{f}, \sigma \in \mathfrak{S}_{\{2 f+1,2 f+2, \cdots, n\}}\right\} .
$$

Then $\mathrm{Bd}^{(f)}\left(d_{1} ; n\right)$ consists of all the Brauer $n$-diagrams whose top horizontal edges are

$$
\left((1) d_{1},(2) d_{1}\right),\left((3) d_{1},(4) d_{1}\right), \cdots,\left((2 f-1) d_{1},(2 f) d_{1}\right) .
$$

The following result can be proved in the same way as Lemma 4.4. 
Lemma 4.5 Let $f$ be an integer with $0 \leq f \leq[n / 2]$. Let $d_{1} \in \mathfrak{D}_{f}$. Then for any $\sigma \in \mathfrak{S}_{n}$,

$$
\left(\sum_{D \in \mathrm{Bd}^{(f)}\left(d_{1} ; n\right)} D\right) \sigma=\sum_{D \in \mathrm{Bd}^{(f)}\left(d_{1} ; n\right)} D .
$$

Let $\underline{i}=\left(i_{1}, i_{2}, \cdots, i_{n}\right)$, where $1 \leq i_{j} \leq 2 m$ for each $j$. An ordered pair $(s, t)(1 \leq$ $s<t \leq n)$ is called a symplectic pair in $\underline{i}$ if $i_{s}=i_{t}^{\prime}$. Two ordered pairs $(s, t)$ and $(u, v)$ are called disjoint if $\{s, t\} \cap\{u, v\}=\emptyset$. We define the symplectic length $\ell_{s}(\underline{i})$ to be the maximal number of disjoint symplectic pairs $(s, t)$ in $\underline{i}$. Let

$$
I(2 m, n):=\left\{\underline{i}=\left(i_{1}, \cdots, i_{n}\right) \mid 1 \leq i_{j} \leq 2 m, i_{j} \in \mathbb{N}, \forall j\right\} .
$$

For an arbitrary element $v \in V^{\otimes n}$, we say the simple tensor $v_{\underline{i}}=v_{i_{1}} \otimes \cdots \otimes v_{i_{n}}$ is involved in $v$, if $v_{i}$ has nonzero coefficient in writing $v$ as linear combination $\sum_{\underline{j} \in I(2 m, n)} k_{\underline{j}} v_{j}$ of the basis $\left\{v_{\underline{j}} \mid \underline{j} \in I(2 m, n)\right\}$ of $V^{\otimes n}$. We now consider a special case of Lemma 3.2.

Proposition 4.6 We have that

$$
\sum_{D \in \mathrm{Bd}_{n}(m+1, m+1)} D \in \operatorname{Ker} \varphi .
$$

Proof By the above discussion and the definition of $\mathrm{Bd}_{n}(m+1, m+1)$, any Brauer diagram $D \in \mathrm{Bd}_{n}(m+1, m+1)$ only acts on the first $m+1$ components of any simple $n$-tensor $v_{i_{1}} \otimes v_{i_{2}} \otimes \cdots \otimes v_{i_{n}} \in V^{\otimes n}$. Therefore, to show that $\sum_{D \in \mathrm{Bd}_{n}(m+1, m+1)} D \in$ $\operatorname{Ker} \varphi$, we can assume without loss of generality that $n=m+1$. Note that

$$
\begin{aligned}
\sum_{D \in \mathrm{Bd}_{n}(m+1, m+1)} D & =\sum_{0 \leq f \leq[n / 2]} \sum_{d_{2} \in \mathfrak{D}_{f}} \sum_{D \in \mathrm{Bd}^{(f)}\left(n ; d_{2}\right)} D \\
& =\sum_{0 \leq f \leq[n / 2]} \sum_{d_{1} \in \mathfrak{D}_{f}} \sum_{D \in \mathrm{Bd}^{(f)}\left(d_{1} ; n\right)} D .
\end{aligned}
$$

Suppose that $\sum_{D \in \mathrm{Bd}_{n}(m+1, m+1)} D \notin \operatorname{Ker} \varphi$. Then there exists a simple $n$-tensor $v_{\underline{i}}=$ $v_{i_{1}} \otimes v_{i_{2}} \otimes \cdots \otimes v_{i_{n}} \in V^{\otimes n}$, such that

$$
v_{\underline{i}} \sum_{D \in \operatorname{Bd}_{n}(m+1, m+1)} D \neq 0 .
$$

Suppose that $\ell_{s}(\underline{i})=f$ for some integer $0 \leq f \leq[n / 2]$. By Lemma 4.4 , for any $\sigma \in \mathfrak{S}_{n}$,

$$
\sigma^{-1} \sum_{D \in \operatorname{Bd}_{n}(m+1, m+1)} D=\sum_{D \in \operatorname{Bd}_{n}(m+1, m+1)} D .
$$

Therefore, replacing $v_{\underline{i}}$ by $v_{\underline{i} \sigma}$ if necessary, we can assume without loss of generality that $i_{2 s-1}=i_{2 s}^{\prime}<i_{2 s}$ for each integer $1 \leq s \leq f, i_{1} \leq i_{3} \leq \cdots \leq i_{2 f-1}$ and $i_{2 f+1} \leq$ 
$i_{2 f+2} \leq \cdots \leq i_{n}$. Furthermore, if $i_{j}=i_{k}$ for some integers $j \neq k$, then

$$
v_{\underline{i}} \sum_{D \in \operatorname{Bd}_{n}(m+1, m+1)} D=v_{\underline{i}} \frac{1+s_{(j, k)}}{2} \sum_{D \in \mathrm{Bd}_{n}(m+1, m+1)} D=0,
$$

where $s_{(j, k)}$ denotes the transposition $(j, k)$ in $\mathfrak{S}_{n}$, and we have used the fact that the length of $s_{(j, k)}$ is an odd integer. Therefore, we can deduce that $i_{1}, i_{2}, \cdots, i_{n}$ are pairwise distinct. Hence, $i_{2 s-1}=i_{2 s}^{\prime}<i_{2 s}$ for each integer $1 \leq s \leq f, i_{1}<i_{3}<\cdots<$ $i_{2 f-1}$ and $i_{2 f+1}<i_{2 f+2}<\cdots<i_{n}$. Since $n=m+1, i_{1}, i_{2}, \cdots, i_{n}$ are pairwise distinct implies that $f$ must be bigger than 0 . Hence $0<f \leq[n / 2]$.

Note that if $g>f$ and $D \in \operatorname{Bd}^{(g)}\left(d_{1}, n\right)$, then $v_{i} D=0$. Hence we get that

$$
v_{\underline{i}} \sum_{0 \leq g \leq f} \sum_{d_{1} \in \mathfrak{D}_{g}} \sum_{D \in \mathrm{Bd}^{(g)}\left(d_{1} ; n\right)} D \neq 0 .
$$

By Lemma 4.5, it is easy to see that for any $\sigma \in \mathfrak{S}_{n}$,

$$
v_{\underline{i}} \sum_{0 \leq g \leq f} \sum_{d_{1} \in \mathfrak{D}_{g}} \sum_{D \in \mathrm{Bd}^{(g)}\left(d_{1} ; n\right)} D \sigma=v_{\underline{i}} \sum_{0 \leq g \leq f} \sum_{d_{1} \in \mathfrak{D}_{g}} \sum_{D \in \mathrm{Bd}^{(g)}\left(d_{1} ; n\right)} D .
$$

Given any simple $n$-tensor $v_{b}:=v_{b_{1}} \otimes \cdots \otimes v_{b_{n}}$ which is involved in the left-hand side of (4.2), we claim that $\bar{\ell}_{s}(\underline{b})=f$ and $b_{1}, \cdots, b_{n}$ are pairwise distinct. In fact, since $\ell_{s}(\underline{i})=f$, it is clear that $\ell_{s}(\underline{b})=f$. Suppose that $b_{j}=b_{k}$ for some integers $j \neq k$ and $v_{\underline{b}}$ appears with coefficient $A_{\underline{b}} \neq 0$ in the expansion of the left-hand side of (4.2), then $v_{\underline{b}}$ also appears with coefficient $A_{\underline{b}} \neq 0$ in the expansion of

$$
v_{\underline{i}} \sum_{0 \leq g \leq f} \sum_{d_{1} \in \mathfrak{D}_{g}} \sum_{D \in \mathrm{Bd}^{(g)}\left(d_{1} ; n\right)} D s_{(j, k)}=v_{\underline{i}} \sum_{0 \leq g \leq f} \sum_{d_{1} \in \mathfrak{D}_{g}} \sum_{D \in \mathrm{Bd}^{(g)}\left(d_{1} ; n\right)} D .
$$

As a result, $v_{\underline{b}}$ also appears with coefficient $A_{\underline{b}} \neq 0$ in the expansion of

$$
v_{\underline{i}} \sum_{0 \leq g \leq f} \sum_{d_{1} \in \mathfrak{D}_{g}} \sum_{D \in \mathrm{Bd}^{(g)}\left(d_{1} ; n\right)} D\left(1+s_{(j, k)}\right) / 2,
$$

which is impossible, since

$$
\left(v_{b_{1}} \otimes \cdots \otimes v_{b_{n}}\right)\left(1+s_{(j, k)}\right) / 2=0 .
$$

By (4.3), for any $\sigma \in \mathfrak{S}_{n}, v_{\underline{b}}$ is involved in the left-hand side of (4.2) if and only if $v_{b \sigma}$ is involved in the left-hand side of (4.2). Therefore, we can choose a simple $n$-tensor $v_{b}$ which is involved in the expansion of the left-hand side of (4.2) such that $b_{2 s-1}=b_{2 s}^{\prime}<b_{2 s}$ for each integer $1 \leq s \leq f, b_{1}<b_{3}<\cdots<b_{2 f-1}$, and $b_{1}, \cdots, b_{n}$ are pairwise distinct. We now consider the intersection of indices in $\underline{i}$ and $\underline{b}$. By Remark 4.3, the symplectic pairs in $\underline{b} \backslash \underline{i}$ must be produced through the action of contraction operators (i.e., horizontal edges in top row), while the symplectic pairs in $\underline{b} \cap \underline{i}$ may be produced through either the action of contraction operators or the permutation action of vertical edges. Therefore, by the argument at the beginning of 
this paragraph, we can further require that there exists an integer $0 \leq r \leq f$ such that:

(a) $b_{t}=i_{t}$ for each integer $2 r+1 \leq t \leq n$, and

(b) $\left\{b_{1}, b_{2}, \cdots, b_{2 r}\right\} \cap\left\{i_{1}, i_{2}, \cdots, i_{n}\right\}=\emptyset$.

Since $n=m+1$, the above conditions and the fact that $i_{1}, \cdots, i_{n}$ are pairwise distinct imply that $r$ must be less than $f$. Hence $0 \leq r<f$.

Let $g$ be an integer with $0 \leq g \leq f, d_{1} \in \mathfrak{D}_{g}, D \in \operatorname{Bd}^{(g)}\left(d_{1} ; n\right)$, where

$$
D=d_{1}^{-1} e_{1} e_{3} \cdots e_{2 g-1} \sigma d_{2}, \sigma \in \mathfrak{S}_{\{2 g+1,2 g+2, \cdots, n\}}, d_{2} \in \mathfrak{D}_{g} .
$$

We claim that $v_{\underline{b}}$ appears with nonzero coefficient in the expansion of $v_{\underline{i}} D$ if and only if

(1) $g \geq r, \sigma=1$, and

(2) the horizontal edges in the top row of $D$ are of the form

$$
\begin{gathered}
(1,2),(3,4), \cdots,(2 r-1,2 r),\left(2 a_{1}-1,2 a_{1}\right),\left(2 a_{2}-1,2 a_{2}\right), \cdots, \\
\left(2 a_{g-r}-1,2 a_{g-r}\right),
\end{gathered}
$$

where $a_{1}, \cdots, a_{g-r}$ are some integers satisfying $r+1 \leq a_{1}<a_{2}<\cdots<a_{g-r} \leq$ $f$, and

(3) the horizontal edges in the bottom row of $D$ is the same as those in the top row of $D$, i.e., $d_{2}=d_{1}$.

In fact, for any Brauer diagram $D$ satisfying the above conditions (1), (2), (3), by Remark 4.3, $v_{b}$ does appear with nonzero coefficient in the expansion of $v_{i} D$, and the coefficient is $(-1)^{g}$; on the other hand, suppose that $v_{\underline{b}}$ appears with nonzero coefficient in the expansion of $v_{i} D$. By our assumption on $\underline{i}$ and $\underline{b}$, it is easy to see that the tensor factor $v_{b_{2 s-1}} \otimes v_{b_{2 s}}$ with $1 \leq s \leq r$ can only be produced through the action of $e_{2 t-1}$ for some $1 \leq t \leq g$. This implies that $g \geq r$. For each integer $j$ with $2 g+1 \leq j \leq n$, by Remark 4.3, the action of $D$ on $v_{i}$ moves the vector in the $j d_{1}$ th position of $v_{\underline{i}}$ (i.e., $\left.v_{i_{j d_{1}}}\right)$ to the $\left(j \sigma d_{2}\right)$ th position. By our assumption on $\underline{i}, \underline{b}$ again, we deduce that $j d_{1}=j \sigma d_{2}$. But by the definition of $\mathfrak{D}_{g}$,

$$
\begin{aligned}
& (2 g+1) d_{1}<(2 g+2) d_{1}<\cdots<(n) d_{1}, \\
& (2 g+1) d_{2}<(2 g+2) d_{2}<\cdots<(n) d_{2} .
\end{aligned}
$$

It follows that $\sigma=1$, and $j d_{1}=j d_{2}$ for any $2 g+1 \leq j \leq n$. Now the remaining statements of our claim follows easily from the fact that $\sigma=1$, our assumption on $\underline{i}$ and $\underline{b}$ as well as Remark 4.3.

Therefore, the coefficient of $v_{\underline{b}}$ in the expansion of

$$
\sum_{d_{1} \in \mathfrak{D}_{g}} \sum_{D \in \mathrm{Bd}^{(g)}\left(d_{1} ; n\right)} v_{\underline{i}} D
$$

is equal to

$$
(-1)^{g}\left(\begin{array}{l}
f-r \\
g-r
\end{array}\right) .
$$


Note that $0 \leq r<f$, it follows that the coefficient of $v_{\underline{b}}$ in the left-hand side of (4.2) is

$$
\sum_{r \leq g \leq f}(-1)^{g}\left(\begin{array}{l}
f-r \\
g-r
\end{array}\right)=0
$$

a contradiction. This completes the proof of the proposition.

Next we consider a more general situation than Proposition 4.6, which is still a special case of Lemma 3.2.

Proposition 4.7 Let $a, b$ be two integers such that $0 \leq a, b \leq n$ and $a+b$ is even. Suppose that $a+b \geq 2 m+2$ and $a \geq b$; then

$$
\sum_{D \in \operatorname{Bd}_{n}(a, b)} D \in \operatorname{Ker} \varphi
$$

Proof By the assumption that $a \geq b$ and the definition of $\operatorname{Bd}_{n}(a, b)$, any Brauer diagram $D \in \operatorname{Bd}_{n}(a, b)$ only acts on the first $a$ components of any simple $n$-tensor in $V^{\otimes n}$. Therefore, we can assume without loss of generality that $n=a$. Also, because of Proposition 4.6, we can assume that $n=a>b$. In particular, $n+b \geq 2 m+2$ and $n=a \geq m+2$. Suppose that $\sum_{D \in \operatorname{Bd}_{n}(n, b)} D \notin \operatorname{Ker} \varphi$. Then there exists a simple $n$-tensor $v_{\underline{i}} \in V^{\otimes n}$ such that

$$
v_{\underline{i}} \sum_{D \in \mathrm{Bd}_{n}(n, b)} D \neq 0 .
$$

Applying Lemma 4.4, we know that for any $\sigma \in \mathfrak{S}_{n}$,

$$
\sigma \sum_{D \in \operatorname{Bd}_{n}(n, b)} D=\sum_{D \in \operatorname{Bd}_{n}(n, b)} D .
$$

Therefore, using the same argument as in the proof of Proposition 4.6, we deduce that $i_{1}, \cdots, i_{n}$ are pairwise distinct. Furthermore, let $f=\ell_{s}(\underline{i})$, we can choose $\underline{i}$ such that $i_{2 s-1}=i_{2 s}^{\prime}<i_{2 s}$ for each integer $1 \leq s \leq f, i_{1}<i_{3}<\cdots<i_{2 f-1}$ and $i_{2 f+1}<i_{2 f+2}<\cdots<i_{n}$.

We define

$$
\Sigma_{f}=\{(1,2),(3,4), \cdots,(2 f-1,2 f)\} .
$$

For each $D \in \operatorname{Bd}_{n}(n, b)$, let $\Sigma^{+}(D)$ be the set of horizontal edges in the top row of $D$. Let $c:=(n-b) / 2$. The assumption (4.4) and the definition of $\operatorname{Bd}_{n}(n, b)$ imply that $f \geq c$. If $f=c$, then (as $i_{1}, \cdots, i_{n}$ are pairwise distinct) we must have $c+b \leq m$, equivalently, $n+b \leq 2 m$, which is a contradiction. Therefore, $f \neq c$. So $c<f \leq$ $[n / 2]$.

By our choice of $\underline{i}$, it is clear that $v_{\underline{i}} D \neq 0$ only if $\Sigma^{+}(D) \subseteq \Sigma_{f}$. Therefore,

$$
v_{\underline{i}} \sum_{D \in \operatorname{Bd}_{n}(n, b)} D=v_{\underline{i}} \sum_{\substack{D \in \operatorname{Bd}_{n}(n, b) \\ \Sigma^{+}(D) \subseteq \Sigma_{f}}} D \neq 0 .
$$


By the definition of $\operatorname{Bd}_{n}(n, b)$ and Remark 4.3, if the simple $n$-tensor $v_{\underline{k}}:=v_{k_{1}} \otimes$ $\cdots \otimes v_{k_{n}}$ is involved in the left-hand side of (4.5), then

$$
k_{b+1}=k_{b+2}^{\prime}, k_{b+3}=k_{b+4}^{\prime}, \cdots, k_{n-1}=k_{n}^{\prime} .
$$

For any $\sigma \in \mathfrak{S}_{b}$, it is easy to see

$$
\sum_{D \in \mathrm{Bd}_{n}(n, b)} D \sigma=\sum_{D \in \operatorname{Bd}_{n}(n, b)} D .
$$

It follows that, for any $\sigma \in \mathfrak{S}_{b}, v_{\underline{k}}$ is involved in the left-hand side of (4.5) if and only if $v_{k \sigma}$ is involved in the left-hand side of (4.5). Therefore, we can choose a simple $n$ tensor $v_{\underline{k}}$ which is involved in the left-hand side of (4.5) such that $k_{2 s-1}=k_{2 s}^{\prime}<k_{2 s}$ for each integer $1 \leq s \leq f-c, k_{2(f-c)+1}<k_{2(f-c)+2}<\cdots<k_{b}$, and $k_{1}, \cdots, k_{b}$ are pairwise distinct. Using the same argument as in the proof of Proposition 4.6, we can further require that there exists an integer $0 \leq r \leq f-c$ such that $k_{t}=i_{t}$ for each integer $2 r+1 \leq t \leq b$, and $\left\{k_{1}, k_{2}, \cdots, k_{2 r}\right\} \cap\left\{i_{1}, i_{2}, \cdots, i_{n}\right\}=\emptyset$. We claim that $r \neq f-c$. In fact, if $r=f-c$, then the above empty intersection condition on $r$ and our assumption on $\underline{i}$ imply that $f-c+n-2 f+f \leq m$, equivalently, $(n+b) / 2 \leq m$, which is impossible. This proves our claim. Hence, $0 \leq r<f-c$.

Let $g$ be an integer with $0 \leq g \leq f, d_{1} \in \mathfrak{D}_{g}, D \in \mathrm{Bd}^{(g)}\left(d_{1} ; n\right)$, where

$$
D=d_{1}^{-1} e_{1} e_{3} \cdots e_{2 g-1} \sigma d_{2}, \sigma \in \mathfrak{S}_{\{2 g+1,2 g+2, \cdots, n\}}, d_{2} \in \mathfrak{D}_{g} .
$$

We claim that $v_{\underline{k}}$ appears with nonzero coefficient in the expansion of $v_{\underline{i}} D$ if and only if

(1) $g \geq r+c, \sigma=1$, and

(2) the horizontal edges in the top row of $D$ are of the form

$$
\begin{gathered}
(1,2),(3,4), \cdots,(2 r-1,2 r),\left(2 a_{1}-1,2 a_{1}\right),\left(2 a_{2}-1,2 a_{2}\right), \cdots, \\
\left(2 a_{g-r}-1,2 a_{g-r}\right),
\end{gathered}
$$

where $a_{1}, \cdots, a_{g-r}$ are some integers satisfying $r+1 \leq a_{1}<a_{2}<\cdots<a_{g-r} \leq$ $f$, and

(3) the horizontal edges in the bottom row of $D$ are of the form

$$
\begin{gathered}
(1,2),(3,4), \cdots,(2 r-1,2 r),\left(2 a_{1}-1,2 a_{1}\right),\left(2 a_{2}-1,2 a_{2}\right), \cdots, \\
\left(2 a_{g-c-r}-1,2 a_{g-c-r}\right),(b+1, b+2),(b+3, b+4), \\
\cdots,(n-1, n) .
\end{gathered}
$$

In fact, for any Brauer diagram $D$ satisfying the above conditions (1), (2), (3), by Remark 4.3, $v_{\underline{k}}$ does appear with nonzero coefficient in the expansion of $v_{\underline{i}} D$, and the coefficient is

$$
\left(\prod_{s=1}^{c} \epsilon_{k_{b+2 s-1}^{\prime}}, k_{b+2 s-1}\right)(-1)^{g-c} .
$$


On the other hand, suppose that $v_{\underline{k}}$ appears with nonzero coefficient in the expansion of $v_{\underline{i}} D$. By our assumption on $\underline{i}$ and $\underline{k}$ and the definition of $\operatorname{Bd}_{n}(n, b)$, it is easy to see that the tensor factor $v_{k_{2 s-1}} \otimes v_{k_{2 s}}$ with $1 \leq s \leq r$ and $v_{k_{b+2 t-1}} \otimes v_{k_{b+2 t}}$ with $1 \leq t \leq c$ can only be produced through the action of $e_{2 t-1}$ for some $1 \leq t \leq g$. This implies that $g \geq r+c$. For each integer $j$ with $2 g+1 \leq j \leq n$, by Remark 4.3, the action of $D$ on $v_{i}$ moves the vector in the $j d_{1}$ th position of $v_{\underline{i}}$ (i.e., $v_{i_{j d_{1}}}$ ) to the $\left(j \sigma d_{2}\right)$ th position. By our assumption on $\underline{i}, \underline{k}$ again, we deduce that $j d_{1}=j \sigma d_{2}$. But by the definition of $\mathfrak{D}_{g}$,

$$
\begin{aligned}
& (2 g+1) d_{1}<(2 g+2) d_{1}<\cdots<(n) d_{1}, \\
& (2 g+1) d_{2}<(2 g+2) d_{2}<\cdots<(n) d_{2} .
\end{aligned}
$$

It follows that $\sigma=1$, and $j d_{1}=j d_{2}$ for any $2 g+1 \leq j \leq n$. Now the remaining statements of our claim follows easily from the fact that $\sigma=1$, our assumption on $\underline{i}$ and $\underline{k}$ as well as Remark 4.3.

Therefore, the coefficient of $v_{\underline{b}}$ in the expansion of

$$
\sum_{d_{1} \in \mathfrak{D}_{g}} \sum_{D \in \mathrm{Bd}^{(g)}\left(d_{1} ; n\right)} v_{\underline{i}} D
$$

is equal to

$$
\left(\prod_{s=1}^{c} \epsilon_{k_{b+2 s-1}^{\prime}}, k_{b+2 s-1}\right)(-1)^{g-c}\left(\begin{array}{l}
f-r \\
g-r
\end{array}\right) .
$$

Note that $0 \leq r<f-c$, it follows that the coefficient of $v_{\underline{b}}$ in the left-hand side of (4.2) is

$$
\left(\prod_{s=1}^{c} \epsilon_{k_{b+2 s-1}^{\prime}, k_{b+2 s-1}}\right) \sum_{c+r \leq g \leq f}(-1)^{g-c}\left(\begin{array}{l}
f-r \\
g-r
\end{array}\right)=0,
$$

a contradiction. This completes the proof of the proposition.

Finally, thanks to Proposition 4.7, to complete the proof of Lemma 3.2, we only need to prove the following proposition. We are grateful to the referee for providing the following simple proof.

Proposition 4.8 Let $a, b$ be two integers such that $0 \leq a, b \leq n$ and $a+b$ is even. Suppose that $a+b \geq 2 m+2$ and $b \geq a$; then

$$
\sum_{D \in \operatorname{Bd}_{n}(a, b)} D \in \operatorname{Ker} \varphi
$$

Proof Given a Brauer $n$-diagram $D$, we define $\bar{D}$ by reflecting $D$ top to bottom. For example, let $D$ be the following Brauer 4-diagram: 


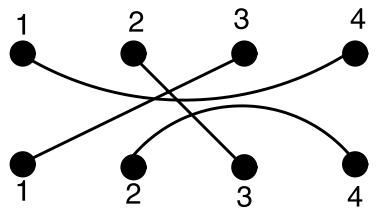

then $\bar{D}$ is the following Brauer 4-diagram:

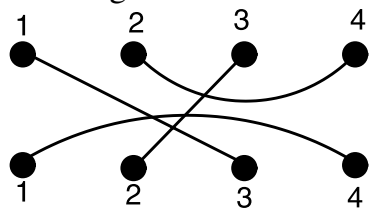

Extending this linearly to the whole of the Brauer algebra, and it gives an antiautomorphism of the Brauer algebra.

Given this automorphism, we have a contravariant duality on modules: if $M$ is a module for the Brauer algebra $\mathfrak{B}_{n}(-2 m)_{\mathbb{C}}$, then the dual space $M^{*}$ is also a module, with

$$
(f d)(m):=f(m \bar{d}),
$$

for $f \in M^{*}, m \in M, d \in \mathfrak{B}_{n}(-2 m) \mathbb{C}$. For each integer $i$ with $1 \leq i \leq 2 m$, let $v_{i}^{*} \in V^{*}$ be defined by $v_{i}^{*}\left(v_{j}\right)=\delta_{i, j}, \forall 1 \leq j \leq 2 m$. For any simple $n$-tensor $v_{i}=v_{i_{1}} \otimes \cdots \otimes$ $v_{i_{n}} \in V^{\otimes n}$, let $v_{\underline{i}}^{*}:=v_{i_{1}}^{*} \otimes \cdots \otimes v_{i_{n}}^{*} \in\left(V^{*}\right)^{\otimes n}$. We identify $\left(V^{*}\right)^{\otimes n}$ with $\left(V^{\otimes n}\right)^{*}$ in a natural way, i.e., such that

$$
v_{\underline{i}}^{*}\left(v_{\underline{j}}\right)=\delta_{\underline{i}, \underline{j}}, \forall \underline{i}, \underline{j} \in I(2 m, n)
$$

We claim that the map

$$
\theta: \sum_{\underline{i} \in I(2 m, n)} a_{\underline{i}} v_{\underline{i}} \mapsto \sum_{\underline{i} \in I(2 m, n)} a_{\underline{i}} v_{\underline{i}}^{*}, \forall a_{\underline{i}} \in \mathbb{C},
$$

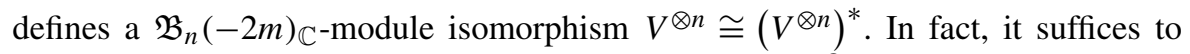
check $\theta\left(v_{\underline{i}} x\right)=\theta(x) v_{\underline{i}}$ for each simple $n$-tensor $v_{\underline{i}} \in V^{\otimes n}$ and each generator $x$ of $\mathfrak{B}_{n}(-2 m)_{\mathbb{C}}$, which is actually trivial. As a result, we get that for any $D \in \mathrm{Bd}_{n}$, $\underline{i}, \underline{j} \in I(2 m, n)$, the coefficient of $v_{i}$ in $v_{j} D$ is the same as the coefficient of $v_{j}$ in $v_{i} \bar{D}$. Therefore, $\operatorname{Ker} \varphi=\overline{\operatorname{Ker} \varphi}$. Now applying Proposition 4.7, we deduce that $\sum_{D \in \mathrm{Bd}_{n}(a, b)} D \in \operatorname{Ker} \varphi$. This completes the proof of the proposition.

\section{References}

1. Atiyah, M.F., Macdonald, I.G.: Introduction to Commutative Algebra. Wesley, Reading (1969)

2. Brauer, R.: On algebras which are connected with semisimple continuous groups. Ann. Math. 38, 857-872 (1937)

3. Brown, W.P.: An algebra related to the orthogonal group. Michigan Math. J. 3, 1-22 (1955-1956)

4. Brown, W.P.: The semisimplicity of $\omega_{f}^{n}$. Ann. Math. 63, 324-335 (1956) 
5. Benkart, G., Chakrabarti, M., Halverson, T., Leduc, R., Lee, C., Stroomer, J.: Tensor product representations of general linear groups and their connections with Brauer algebras. J. Algebra 166, 529-567 (1994)

6. Birman, J., Wenzl, H.: Braids, link polynomials and a new algebra. Trans. Am. Math. Soc. 313(1), 249-273 (1989)

7. De Concini, C., Procesi, C.: A characteristic free approach to invariant theory. Adv. Math. 21, 330-354 (1976)

8. Carter, R.W., Lusztig, G.: On the modular representations of general linear and symmetric groups. Math. Z. 136, 193-242 (1974)

9. Chari, V., Pressley, A.: A Guide to Quantum Groups. Cambridge University Press, Cambridge (1994)

10. Dipper, R., Doty, S.: The rational Schur algebra. Preprint math.RT/0511663 (2005)

11. Dipper, R., Doty, S., Hu, J.: Brauer algebras, symplectic Schur algebras and Schur-Weyl duality. Trans. Am. Math. Soc. 360, 189-213 (2008)

12. Dipper, R., James, G.D.: Representations of Hecke algebras of general linear groups. Proc. Lond. Math. Soc. 52(3), 20-52 (1986)

13. Dipper, R., James, G.D.: Blocks and idempotents of Hecke algebras of general linear groups. Proc. Lond. Math. Soc. 54(3), 57-82 (1987)

14. Du, J., Parshall, B., Scott, L.: Quantum Weyl reciprocity and tilting modules. Commun. Math. Phys. 195, 321-352 (1998)

15. Doty, S.: Polynomial representations, algebraic monoids, and Schur algebras of classic type. J. Pure Appl. Algebra 123, 165-199 (1998)

16. Enyang, J.: Cellular bases for the Brauer and Birman-Murakami-Wenzl algebras. J. Algebra 281, 413-449 (2004)

17. Fishel, S., Grojnowski, I.: Canonical bases for the Brauer centralizer algebra. Math. Res. Lett. 2(1), 15-26 (1995)

18. Graham, J.J., Lehrer, G.I.: Cellular algebras. Invent. Math. 123, 1-34 (1996)

19. Grigor'ev, D.Ju.: An analogue of the Bruhat decomposition for the closure of the cone of a Chevalley group of the classical series. Sov. Math. Dokl. 23, 393-397 (1981)

20. Härterich, M.: Murphy bases of generalized Temperley-Lieb algebras. Arch. Math. 72(5), 337-345 (1999)

21. Hu, J.: Quasi-parabolic subgroups of the Weyl group of type D. Eur. J. Comb. 28(3), 807-821 (2007)

22. Hanlon, P., Wales, D.B.: On the decomposition of Brauer's centralizer algebras. J. Algebra 121, 409445 (1989)

23. Hanlon, P., Wales, D.B.: Eigenvalues connected with Brauer's centralizer algebras. J. Algebra 121, 446-475 (1989)

24. Jimbo, M.: A $q$-analogue of $U(\mathfrak{g l}(N+1))$, Hecke algebras, and Yang-Baxter equation. Lett. Math. Phys. 11, 247-252 (1986)

25. Künzer, M., Mathas, A.: Elementary divisors of Specht modules. Eur. J. Comb. 26, 943-964 (2005)

26. Koike, K., Terada, I.: Young-diagrammatic methods for the representation theory of the classical groups of type $B_{n}, C_{n}, D_{n}$. J. Algebra 107, 466-511 (1987)

27. Macdonald, I.G.: Symmetric Functions and Hall Polynomials, Oxford Mathematical Monographs. Clarendon Oxford University Press, New York (1979)

28. Mathas, A.: Iwahori-Hecke Algebras and Schur Algebras of the Symmetric Group, University Lecture Series, vol. 15. American Mathematical Society, Providence, R.I. (1999)

29. Murakami, J.: The Kauffman polynomial of links and representation theory. Osaka J. Math. 26(4), 745-758 (1987)

30. Murphy, E.: On the representation theory of the symmetric groups and associated Hecke algebras. J. Algebra 152, 492-513 (1992)

31. Murphy, E.: The representations of Hecke algebras of type $A_{n}$. J. Algebra 173, 97-121 (1995)

32. Oehms, S.: Centralizer coalgebras, FRT-construction, and symplectic monoids. J. Algebra 244(1), 19-44 (2001)

33. Schur, I.: Über die rationalen Darstellungen der allgemeinen linearen Gruppe (1927). Reprinted in I. Schur, Gesammelte Abhandlungen, vol. III, pp. 68-85. Springer, Berlin (1973)

34. Weyl, H.: The Classical Groups, Their Invariants and Representations. Princeton University Press, Princeton (1946)

35. Wenzl, H.: On the structure of Brauer's centralizer algebras. Ann. Math. 128, 173-193 (1988) 